\section{CURRENT DEVELOPMENT OF SORBENTS DERIVED FROM PLANT AND ANIMAL WASTE AS GREEN SOLUTION FOR TREATING POLLUTED AQUEOUS MEDIA}

\author{
Sri Martinia*, Sharmeen Afrozeb
}

aUniversitas Muhammadiyah Palembang, Indonesia bCurtin University Australia, Australia
Article history

Received

27 June 2021

Received in revised form

14 September 2021

Accepted

23 September 2021

Published Online

20 October 2021

*Corresponding author sri_martini@um-palembang.ac.id

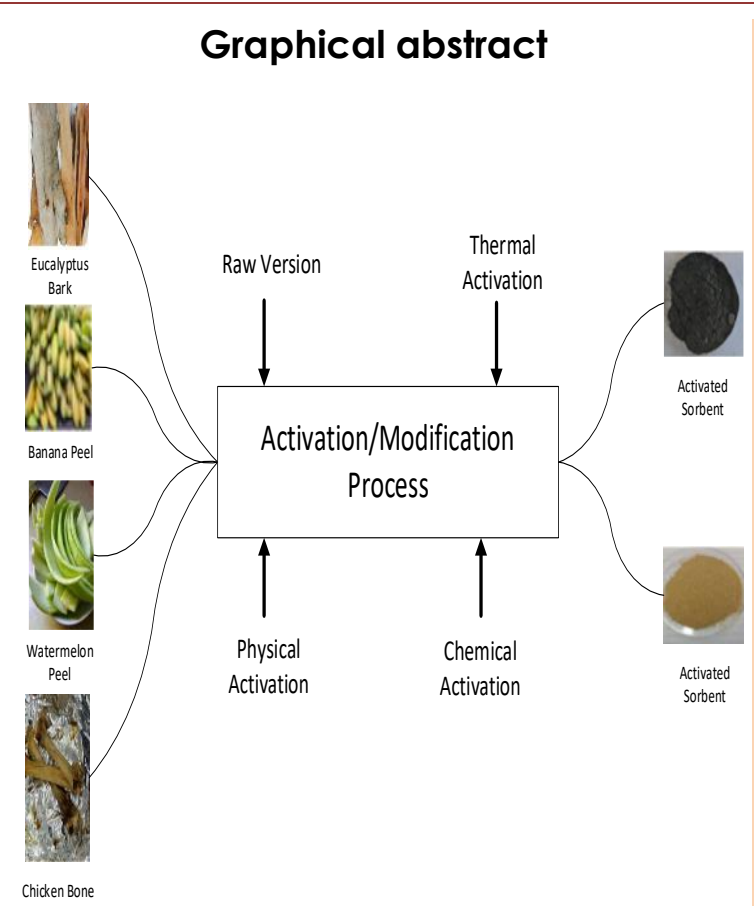

\begin{abstract}
In this review article, the recent development of organic sorbents derived from plant and animal waste for treating polluted aqueous media is presented. The application of biomass has been recognized reliable to adsorb hazardous pollutants contained in wastewater generated by emerging industries. This paper also discusses other existing technologies for removing pollutants including heavy metals, dyes, oil and grease, antibiotic and phenolic compounds from polluted aqueous media. Furthermore, several prominent examples of plant and animal wastes such as eucalyptus bark, pine bark, rice husk, various fruit peels, and animal bones are also properly reviewed. The effects of modification techniques on sorbent performance such as carbonization, pyrolysis, and chemical impregnation using acid or alkaline chemicals are considerably included. Some gaps in current literature are also discussed including the tendency of certain targeted pollutants, the use of simulated aqueous media, the scale of research projects, and the prominent modification procedures. Eventually, based on the relevant literature, clear conclusion has been drawn that these natural disposals have great potentiality as environmentally friendly sorbent alternative to other commercial expensive sorbents, and their reuse has been part of green solutions supporting a circular economy system.
\end{abstract}

Keywords: Adsorption, sorbent, plant waste, animal waste, pollutants

(c) 2021 Penerbit UTM Press. All rights reserved

\subsection{INTRODUCTION}

Polluted aqueous media derived from various industrial sources contain harmful pollutants impacting environment and human health [1, 2]. Without proper treatment and precaution steps, this issue can also lead to clean water scarcity for human needs. Therefore, decent treatment for this polluted water is compulsory approach that can both improve water quality and avert other negative consequences [3].
To date, more research has focused on minimizing the threats posed by pollutants in order to avoid their harmful effects [4, 5]. The increasing number of industrial sectors globally has generated the amount of wastewater containing organic and inorganic pollutants such as oil and grease, heavy metals, dyes, phenolic compounds, antibiotic compounds, suspended and dissolved solids, and etc. Petroleum refinery and petrochemical industries along with battery manufacturing, textiles, tanneries, plastics, electroplating, pharmaceuticals, cosmetics, pulp and 
paper, paints, and edible oil production have been linked to the emerging industrial sectors producing damaging pollutants aforementioned $[5,6]$.

It is argued that polluted wastewaters derived from different manufacturing processes may have their own characters. Textile effluent, for example, has high concentration of inorganic dyes and chemical oxygen demand (COD) along with high loading of various mutagenic heavy metals [7] while olive oil mill or petroleum refinery wastewaters mostly contains high phenol, oil grease and particular heavy metals $[8,9]$.

In order to overcome these matters, several treatment techniques including ion exchange, adsorption, filtration, membrane filtration, membrane bioreactor, sedimentation, electro dialysis, advanced oxidation processes, biological, electrochemical process or integrated treatment system are available and can be properly selected to separate targeted pollutants from the solution [10-13]. However, each of those techniques has its own advantageous and disadvantageous features. When it comes to consider some aspects like lower energy consumption, simple operation, high efficiency, and environmentally friendly behavior, adsorption can still be regarded as one of the best methods [14, 15].

Currently, there are various sorbent types that have been investigated and produced from various precursors like metal oxide, organic carbon-based materials, or metal organic compounds $[2,5,16]$. The increasing interest in circular economic perspective by recycling the wastes for keeping the quality of environment has also triggered more research on the further usage of assorted organic based materials [17]. On the other flip side, the increasing global population has resulted in the increase in human needs related to food and energy sources. As a consequence, there have been more undesirable byproducts and biomass residue. This phenomena bring more challenges for adapting and improving the concept of circular economy society. Therefore, there is a strong relationship between waste management and wastewater treatment to circular economy system [18].

Even though several articles already reviewed the application of natural-based materials, to the best of our knowledge, specific discussion on the usage of activated sorbent derived from plant and animal wastes for treating polluted aqueous media along with their comparative performance is still needed. Therefore, this review article corroborates the relatively recent application of both types of biomass for pollutants removal from simulated and raw industrial effluents along with the characters and sources of several prominent pollutants.

\subsection{WATER TREATMENT TECHNIQUE}

Water and wastewater treatment technology can be applied in a sole or an integrated system. An integrated system combines two or more treatment methods depending on several measurements such as the initial quality, expected outcome, and final purposes of treated water and wastewater [19]. Therefore, this work will also present some available technologies that can be implemented for treating water and wastewater before focusing the discussion on current research regarding the utilization of various plant and animal-based sorbent.

\subsection{Membrane Separation}

Membrane technology can produce permeate or treated water with high purity degree as it is able to barrier a wide variety of pollutants regarding their size or molecular weight $[20,21]$. Other familiar benefits of this technique are its practicality and flexibility in the operation process. However, considerable operational and maintenance cost have to be assessed properly to conduct some strategies for avoiding severe fouling on membrane surface due to trapped pollutants on membrane pores through cleaning stage, chemical surface modification, or pre-treatment process $[22,23]$. The mechanism of membrane fouling may follow one of Hermia's models that consist of four different patterns namely complete blocking, standard blocking, intermediate blocking, and cake filtration models [11, 24].

\subsection{Advanced Oxidation Processes}

Advanced oxidation processes (AOPs) utilize oxidative agents to produce hydroxyl radicals promoting organic compounds mineralization and transforming them into water, carbon dioxide, and other harmless products $[25,26]$. Several techniques can be chosen to perform oxidation process including Fenton, Fenton-like, photo assisted Fenton and photocatalytic reaction [25-28]. However, apart from the practicality and effectiveness, AOPs methods have some drawbacks related to significant chemicals use, sludge generation, and by-product formation [19].

\subsection{Biological Process}

Biological processes utilize microorganisms including fungi, bacteria, yeast, and algal biomass for reducing harmful pollutants in solution [29-31]. It mostly needs longer reaction time and wider treatment place. However, it is not only safe for environment, but also having good removal efficiency and lower operational cost [32].

\subsection{Adsorption}

Adsorption is notable for its satisfactory outcome along with high removal efficiency, less energy and chemicals consumption. This system attracts pollutants from polluted aqueous media to be accumulated on the active sites of sorbent surface 
[5]. Particular pollutants and sorbent properties including surface or pore diffusion, electrostatic interaction, hydrogen bonding, and van der walls forces as well as chosen operating conditions are some dominant factors affecting adsorption process $[33,34]$. Several activation techniques such as carbonization, chemical activation, and pyrolysis can also be conducted to increase the adsorption capacity of the sorbent prior to adsorption process (Figure 1) [35-37].

Moreover, some carbon-based sorbents have been utilized in terms of the source of carbon precursors as well as final product structures such as activated carbon (AC), biochar, carbon nanotubes (CNTs), graphene, and graphene oxides. An increasing interest in using natural organic wastes as the main source of sorbent material then has been significantly recognized due to their reliable availability, a wide range of choices, and high biodegradability as well as cost-effectiveness [5, 16, 38].

\subsubsection{Adsorption Isotherm Models}

The interaction process between sorbent and attracted pollutants was affected by the affinity of the sorbent taking place until the establishment of equilibrium condition. Therefore, it is crucial to study the quantification of their interaction as expressed by batch equilibrium isotherm. In general, there are two well-known isotherm models available to understand the adsorption mechanism like Freudlich and Langmuir [39]. While Freundlich isotherm model believed that the adsorption process solely come about on the heterogeneous sorbent surface with unequal sites with diverse adsorption energies, Langmuir argued that it mostly occurs onto homogeneously uniform sorbent surface having equivalent sites [40]. The linearized formulas (Eqs. 1 and 2) of these isotherm models are tabulated in Table 1.

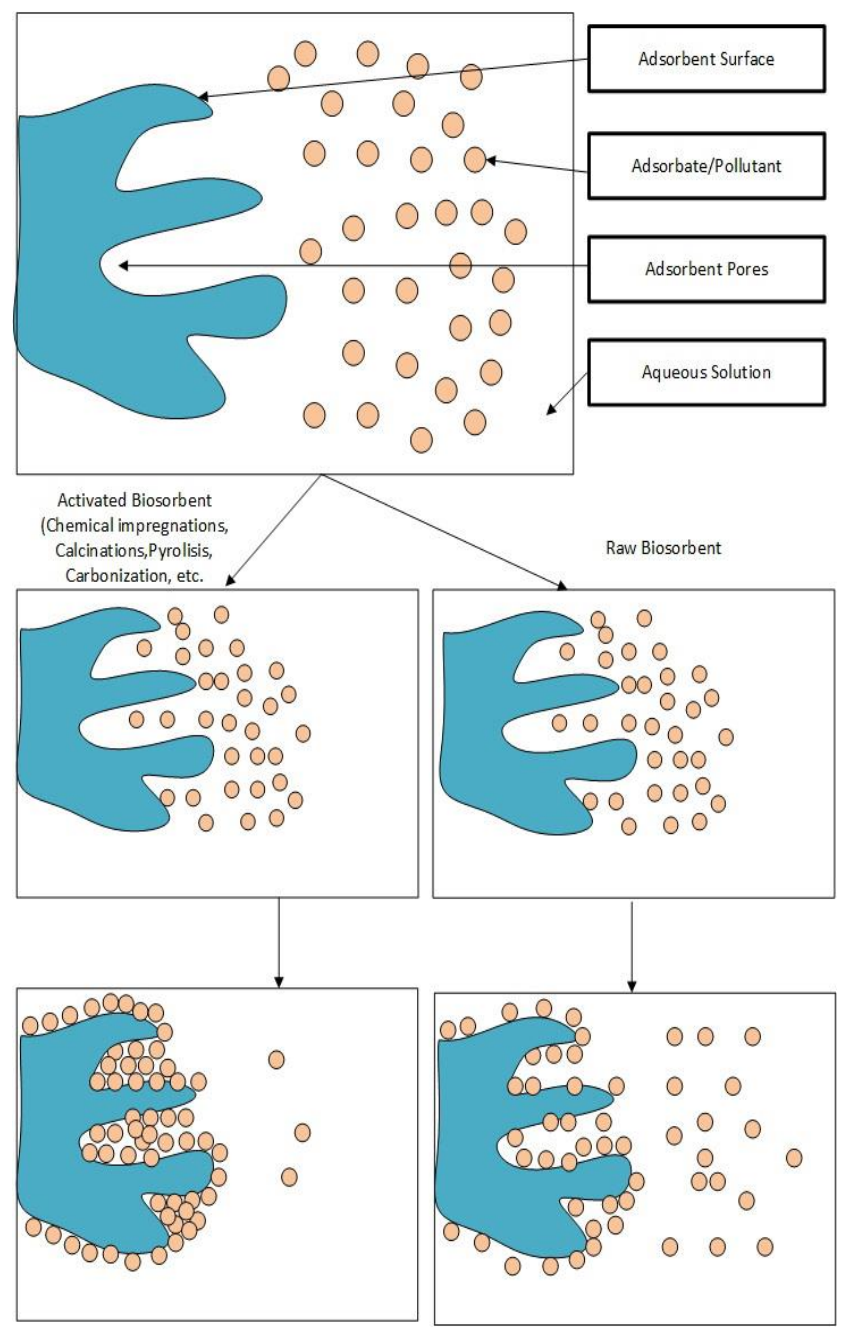

Figure 1 Adsorption process

Table 1 Isotherm models

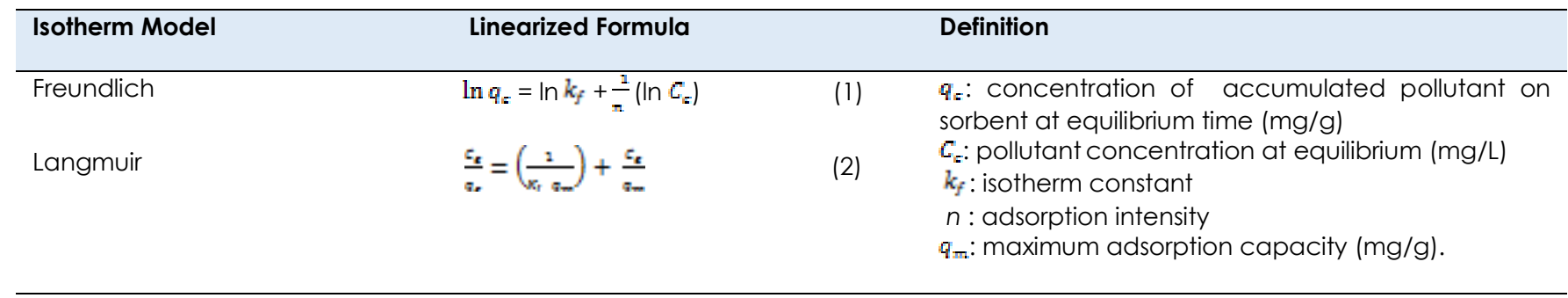

\subsubsection{Adsorption Kinetic Modelling}

Adsorption kinetic analysis can help with understanding the process design for adsorption by predicting the amount of adsorbed pollutants in terms of time parameter along with external mass transfer and solute diffusivity. Kinetic models have been widely used and compared to each other to illustrate the most possible mechanism that happen during adsorption process like pseudo-first order, pseudosecond order, and intra particle diffusion in which their linearized formulas were stated in Eqs. 3-5, respectively (Table 2). Pseudo-first-order model can be regarded to the rate of residence of sites in the 
adsorbent due to adsorption process regarding the proportional rate to the number of void sites while pseudo-second order model proposes the term of reciprocal action between sorbent sites and pollutant particles that may happen due to their robust binding supported by chemisorption process [41, 42]. Additionally, intra-particle diffusion stated different idea by arguing that pollutant uptakes may be inclined to vary proportionally with $t^{1 / 2}$ rather than with contact time [43].

\subsection{Integrated Treatment System}

It is noticed that there is no single treatment provide wastewater purification for complete advantages as there are still some possible drawbacks. Those techniques may have their competence decline at some point of views. Therefore, more research on applying integrated systems which are compatible with each other has to be conducted to overcome the disadvantageous aspects and generate green solutions offering energy-efficient and cost-effective system. Several works have reported promising findings of integrated treatment system in treating synthetic and raw industrial wastewaters. For membrane separation, for instance, severe fouling could be avoided by adapting pre-treatment stage on the membrane feed. As a result, there were much lower fouling rate decline that could bring beneficial effect on membrane usage as a whole including longer membrane life-span and lower maintenance and material changing cost $[11,44,45]$. The integration of two or more different treatment types such as electrocoagulation, ceramic microfiltration membrane, and ozone techniques to purify oily wastewater, for example, has resulted in multiple increase in COD removal efficiency [46]. Other study then supported the benefit of integrated system by reporting the decline of membrane fouling while adopting photocatalytic and adsorption mechanism on the feed before entering membrane system [11].

Table 2 Adsorption kinetics

\begin{tabular}{|c|c|c|c|}
\hline Adsorption Kinetics & Linearized Formula & & Definition \\
\hline Pseudo-first order & $\log \left(q_{z}-q_{t}\right)=\log \left(q_{z}\right)-\frac{k_{1}}{2 \cdot \sin } \cdot t$ & (3) & $\begin{array}{l}q_{\mathrm{t}}: \text { Binded pollutants on sorbent surface } \\
(\mathrm{mg} / \mathrm{g})\end{array}$ \\
\hline Pseudo-second order & $\frac{E}{\sigma_{1}}=\frac{1}{K_{2} a_{k}^{8}}+\frac{1}{\pi_{x}} \cdot t$ & (4) & $\begin{array}{c}K_{2^{x}} \text { The equilibrium rate constant }\left(\mathrm{min}^{-1}\right) \\
t: \text { Contact time (min) } \\
\left.k_{2}: \text { Order rate constant ( } / \mathrm{mg} \mathrm{min}\right)\end{array}$ \\
\hline Intra-particle diffusion & $q_{t}=K_{i d: ~} t^{a s}$ & (5) & $\begin{array}{l}K_{i 2^{x}} \text { The rate constant, intra-particle diffusion } \\
\text { (mg/g.min }{ }^{0.5)} \\
\text { t0.5: The square root of time }\end{array}$ \\
\hline
\end{tabular}

\subsection{PLANT WASTE BASED-SORBENT}

Research on plant-based sorbent has gained an increasing interest for various purposes. The recycle of abundant plant wastes could be part of green solutions overcoming detrimental issues to the environment related agricultural waste $[47,48]$. Along with this consideration, the need of finding cheaper and more environmentally friendly sorbent materials has led to more efforts to widen the variety of green sorbents utilization [49]. To date, there have been a large number of plant residues such as bark or wood, vegetable leftover, fruit peel and seed, and plant leaves that were reported reliable as sorbent (Table 3).

\subsection{Bark and Wood}

Bark and wood remains are part of renewable sorbent sources abundantly available in nature [50]. Globally, it was estimated that the average volume of wood and above ground woody materials was almost 400 billion cubic meters in the start of the year 2000 , and it would be significantly increasing in year 2020 [51]. Therefore, the availability of this material has provided a great source for creating green and effective sorbents.

\subsubsection{Eucalyptus Bark}

Eucalyptus plant can grow well all seasons especially in certain country such as Australia. Considering its woody waste left worthless on the ground, some studies have then utilized it as the alternative matter for producing sorbent. A report on eucalyptus bark (EB) performance on treating raw petroleum refinery wastewater concluded that the modified EB was more effective to simultaneously remove COD, oil, and $\mathrm{Cr}(\mathrm{III})$ from the effluent than its raw version [10]. The comparative analysis consisting of chemically impregnated EB, carbonized EB, and hybrid activated $E B$ found that a hybrid chemical and thermal activation was useful to significantly boost sorbent efficacy. Other study deployed EB based sorbent for $\mathrm{Cu}$ (II) metal ions removal from raw gold mining wastewater and reported a satisfactory outcome [52]. In this study, raw powder EB put in wastewater with high heavy metals content could result in maximum removal efficiency of CU (II) by around $92 \%$ at $\mathrm{pH} 5$ after $90 \mathrm{~min}$. In another study, extracted eucalyptus grandis lignin was found worth to adsorb 
methylene blue dye from aqueous solution [53]. Its efficacy might be attributed to the presence of several functional groups on the sorbent mass including phenolic hydroxyl and methoxyl groups along with morphological heterogeneity. By having a specific surface area of $20 \mathrm{~m}^{2} / \mathrm{g}$, around $90 \%$ of the dye could be separated from the aqueous media.

\subsubsection{Logwood}

Logwood can be utilized to be a carbonaceous sorbent for adsorbing various pollutants including phenol compounds such as 4-chlorophenol and 4nitrophenol from aqueous media [54]. It was found that phosphoric acid and thermally activated logwood at $500^{\circ} \mathrm{C}$ for $60 \mathrm{~min}$ could form amorphous sorbent having wide surface area, pore volume, and mean pore diameter by $181.49 \mathrm{~m}^{2} / \mathrm{g}, 0.09396 \mathrm{~cm}^{3} / \mathrm{g}$, and $2.07 \mathrm{~nm}$, respectively, resulting in adsorption capacity by $94.09 \mathrm{mg} / \mathrm{g}$ for these phenol compounds. The study then confirmed that the adsorption mechanism of this process fitted well to pseudosecond-order kinetic and Langmuir model where the optimum condition was found at sorbent dosage $20 \mathrm{mg} / \mathrm{L}$ and extremely acidic $\mathrm{pH}$ affected by the solubility and dipolar moment of the phenolic components.

\subsubsection{Pine Bark}

Like other barks, pine bark can also be used and mixed with other substances to create sorbent having excellent performance. A study produced a novel sorbent by combining pine bark matters with graphene particles for removing $\mathrm{Cu}$ (II) metal ion from aqueous solution [55]. It then reported that this mixed sorbent has Langmuir adsorption capacities and BET surface area by $18.4 \mathrm{mg} / \mathrm{g}$ and $484.0 \mathrm{~m}^{2} / \mathrm{g}$, respectively. In contrast, raw pine bark basedsorbent only has $9.2 \mathrm{mg} / \mathrm{g}$ and $378.0 \mathrm{~m}^{2} / \mathrm{g}$ of those parameters. In this case, characteristics change on sorbent surface due to graphene combination must have been a critical function. Furthermore, other report informed that quaternary ammonium-modified pine bark could be employed as sorbent for adsorbing vanadium [56]. They revealed that the initial sodium hydroxide concentration as a determining factor. However, different from other studies which proposed initial modification on organic sorbent, a study reported that modified pine bark sorbent using hydrochloric acid and sodium hydroxide solutions for $\mathrm{CU}(\mathrm{II})$ ions adsorption showed lower uptake than its raw version [57]. Certain factors like operating conditions were then assumed influential affecting the effectiveness of chosen activation method.

\subsection{Banana Waste}

Current studies have reported the potentiality of banana waste as sorbent material especially its peel which can be utilized to remove organic and inorganic pollutants from polluted media. To begin with, methylene blue dye concentration in raw textile wastewater could be reduced by more than $86.88 \%$ using as little as $0.4 \mathrm{~g}$ of banana peel-derived sorbent [47]. It was then followed by other study which created banana peel-based biochar/iron oxide composite to adsorb methylene blue dye. It experienced a great adsorption capacity $862 \mathrm{mg} / \mathrm{g}$ at initial dye concentration $500 \mathrm{mg} / \mathrm{L}$ using near neutral $\mathrm{pH}$ and temperature [58]. Surprisingly, its regeneration study also indicated that this sorbent was economically dependable due to relatively stable performance even after five adsorption cycles.

In terms of metals adsorption like $\mathrm{Cr}(\mathrm{IV})$, activated banana peel-based sorbent studied by Ashraf et al. [59] also showed satisfactory output. Specifically, the report found that maximum $\mathrm{Cr}$ (IV) removal efficiency could be reached up to $88 \%$ and $69 \%$ for acidified banana peel and organo montmorillonite activated banana peel, respectively, both at $\mathrm{pH} 3$ and sorbent dosage $4 \mathrm{~g} / \mathrm{L}$.

\subsection{Water Melon Waste}

Water melon is popular fruit and has a huge amount of peel disposal. Therefore, it is worthy to do recycling on its waste as reported in the thallium(I) adsorption study [60]. The water melon peel-based biochar could reach $178.4 \mathrm{mg} / \mathrm{g}$ of thallium(l) adsorption capacity through ion exchange mechanism. Moreover, metal ions namely $\mathrm{As}(\mathrm{V})$ and $\mathrm{As}(\mathrm{III})$ could also be eliminated using water melon based-sorbent [61]. The comparison study among raw, xanthated, and citric acid-modified watermelon rind-based sorbents proclaimed that xanthated modified water melon rind has higher $A s(V)$ and $A s(I I I)$ removal efficiency. Specifically, compared to its raw version, xanthated watermelon rind has higher adsorption rate by up to $49 \%$ for both metals ions. In addition, watermelon rind activated by employing ZnS nanoparticles for adsorbing various heavy metals named $\mathrm{Pb}(\mathrm{II}), \mathrm{Cd}(\mathrm{II}), \mathrm{CU}(\mathrm{II}), \mathrm{Ni}(\mathrm{II})$ and $\mathrm{Co}(\mathrm{II})$ could also reach substantial loading capacity [62].

\subsection{Rice Husk}

Rice husk potentiality as sorbent material for removing different pollutants has been reported. The typical chemical components of this biomass such as cellulose, hemicellulose, lignin, and other organic matter have been proven favorable supporting adsorption aims [63]. Recalcitrant pollutants like phenol could be removed by rice husk based-sorbent [63]. By thermal activation mode, treated rice husk showed higher surface area leading to higher phenol removal efficiency corresponding to some particular functional groups such as $\mathrm{OH}, \mathrm{C}-\mathrm{C}, \mathrm{C}=\mathrm{C}, \mathrm{C} \equiv \mathrm{C}, \mathrm{C}-\mathrm{O}$. Other than that, the valorization of rice husk by $\mathrm{HCl}$ impregnation for adsorbing various dyes namely direct orange-26, direct red-31, direct blue-67 and direct orange-3GL has been claimed successful, especially when it was compared to rice husk sorbent 
treated by salts and surfactant impregnation [64]. The experimental data showed that acidified rice husk has faster dyes adsorption equilibrium with great regeneration ability.

Furthermore, rice husk-based biochar synthesized using one and two-step $\mathrm{KOH}$-catalysed pyrolysis also showed fruitful output [65]. In this study, biochar with two-step pyrolysis outperformed the other where the sorbent yield decreased with the increase in the mass ratio of $\mathrm{KOH}$ and char. The activation process has a crucial influence on the improvement of both sorbent surface area and carbon porosity. Therefore, biochar derived from the two-step pyrolysis could reach an incredible specific surface area by $2138 \mathrm{~m}^{2} / \mathrm{g}$. This massive micro-porous structure was responsible for adsorbing phenol molecules by getting maximum adsorption capacity $201 \mathrm{mg} / \mathrm{g}$ even in a few minutes of contact time by following Langmuir isotherm and pseudo-second-order kinetic model.

\subsection{Leaves-based Sorbent}

As part of plant materials, leaves can also be deployed as sorbents. There are several studies that have investigated leaves waste including the application of Platanus orientalis leaves through mild hydrothermal carbonization and one-step calcination at temperature $750^{\circ} \mathrm{C}$ [66]. This modified sorbent could attain a great specific surface area by around
$1355.53 \mathrm{~m}^{2} / \mathrm{g}$ useful to remove dyes namely rhodamin $B$ and methylene orange. Furthermore, several species of plant leaves named Magnolia denudate, Magnolia grandiflora and Michelia figo were also investigated for adsorbing methylene blue dye and proved creditable by having maximum adsorption capacity of $185.19 \mathrm{mg} / \mathrm{g}, 149.25 \mathrm{mg} / \mathrm{g}$, and $238.10 \mathrm{mg} / \mathrm{g}$, respectively [67]. Interestingly, their regeneration study still showed satisfying adsorption capacity even after the third cycle.

Other type of leave, birch leave (Betula pendula), activated by sulfuric acid used for adsorbing phenol was also competitive by reaching maximum adsorption capacity of $0.128 \mathrm{mmol} / \mathrm{g}$ [68]. In particular, this study confirmed that best phenol sorption occurred at $35^{\circ} \mathrm{C}$ and $45^{\circ} \mathrm{C}$ for Langmuir's equation. Subsequently, sorbent derived from green neem leave (Azadirachta Indica) used for phenol removal was also inclined to be effective as more than $97.5 \%$ of the targeted pollutant could be pulled out of the solution even though it needed longer contact time (4 h) [69]. Moreover, desorption study by deploying ethanol-treatment showed that the reusability of this matters was around half of its initial content. Eventually, plant leaves named aloe vera and mesquite leave were also reported dependable as sorbent alternative for purging phenol especially at neutral $\mathrm{pH}[70]$.

Table 3 The application of natural sorbents derived from plant wastes

\begin{tabular}{|c|c|c|c|c|c|c|c|c|c|}
\hline Sorbent & $\begin{array}{l}\text { Aqueous } \\
\text { Media }\end{array}$ & $\begin{array}{l}\text { Modification } \\
\text { Technique }\end{array}$ & Pollutant & $\begin{array}{l}\text { Isoterm } \\
\text { Model }\end{array}$ & Kinetics & $\begin{array}{l}\text { Removal } \\
\text { Efficiency } \\
\text { (\%)/ } \\
\text { Adsorption } \\
\text { Capacity } \\
\text { (mg/g) }\end{array}$ & $\begin{array}{l}\text { Optimum } \\
\text { Condition }\end{array}$ & $\begin{array}{l}\text { Desorption } \\
\text { Solution/ } \\
\text { Regenera } \\
\text { tion (\%) }\end{array}$ & Ref. \\
\hline \multirow[t]{3}{*}{$\begin{array}{l}\text { Eucalyp } \\
\text {-tus } \\
\text { bark }\end{array}$} & $\begin{array}{l}\text { Raw } \\
\text { petroleum } \\
\text { refinery } \\
\text { wastewater }\end{array}$ & $\begin{array}{l}\mathrm{H}_{3} \mathrm{PO}_{4} \\
\text { impregnation } \\
\text { / } \\
\text { carbonizatio } \\
\mathrm{n} 400^{\circ} \mathrm{C}\end{array}$ & $\begin{array}{l}\text { COD } \\
\text { Oil } \\
\text { Cr(III) }\end{array}$ & $\begin{array}{l}\text { Freundli- } \\
\text { ch }\end{array}$ & $\begin{array}{l}\text { Intra- } \\
\text { particle } \\
\text { diffusion }\end{array}$ & $\begin{array}{l}80 \% \\
91 \% \\
61 \%\end{array}$ & $\begin{array}{l}\text { Time: } 100 \\
\text { min, dosage: } \\
10 \mathrm{~g} / \mathrm{L}, \mathrm{pH}: 3 \text {, } \\
\mathrm{T}: 25^{\circ} \mathrm{C}\end{array}$ & $\begin{array}{l}\mathrm{NaHCO} 3 \\
(60 \%)\end{array}$ & [10] \\
\hline & $\begin{array}{l}\text { Raw gold } \\
\text { mining } \\
\text { wastewater }\end{array}$ & Drying & $\mathrm{Cu}(\mathrm{II})$ & Langmuir & - & $92 \%$ & $\begin{array}{l}\mathrm{pH}: 5, \mathrm{t}: 90 \\
\mathrm{~min}, \text { dosage: } \\
1.8 \mathrm{~g} / \mathrm{L}\end{array}$ & $\begin{array}{l}0.1 \mathrm{HCl} \\
(87.34 \%)\end{array}$ & {$[52]$} \\
\hline & $\begin{array}{l}\text { Synthetic } \\
\text { solution } \\
\text { Color Index } \\
52015\end{array}$ & $\begin{array}{l}\text { Lignin } \\
\text { extraction+dr } \\
\text { ying }\end{array}$ & $\begin{array}{l}\text { Methyle- } \\
\text { ne blue } \\
\text { dye }\end{array}$ & Langmuir & $\begin{array}{l}\text { Pseudo- } \\
\text { second } \\
\text { order }\end{array}$ & $90 \%$ & $\begin{array}{l}\text { Time: } 480 \\
\text { min, dosage: } \\
225 \text { mg, } \\
\text { stirring: } 150 \\
\text { rpm, pH: } 5.6\end{array}$ & $\begin{array}{l}\text { Milli-Q } \\
\text { water }\end{array}$ & [53] \\
\hline \multirow[t]{3}{*}{$\begin{array}{l}\text { Pine } \\
\text { bark }\end{array}$} & $\begin{array}{l}\text { Synthetic } \\
\text { copper } \\
\text { solution }\end{array}$ & $\begin{array}{l}\text { Mixed with } \\
\text { grapheme/ } \\
\text { pyrolysis } \\
600^{\circ} \mathrm{C}\end{array}$ & $\mathrm{Cu}(\mathrm{II})$ & Langmuir & $\begin{array}{l}\text { Pseudo- } \\
\text { second } \\
\text { order }\end{array}$ & $10.6 \mathrm{mg} / \mathrm{g}$ & $\begin{array}{l}\text { Dosage:0.05 } \\
\text { g, Co: } \\
75 \mathrm{mg} / \mathrm{L} \\
\text { stirring: } 200 \\
\text { rpm, pH 6, } \\
\text { t:24 h, T:25॰C }\end{array}$ & $1 \mathrm{M} \mathrm{HNO}_{3}$ & [55] \\
\hline & $\begin{array}{l}\text { Synthetic } \\
\mathrm{NaVO}_{3} \\
\text { solution }\end{array}$ & $\begin{array}{l}\mathrm{NaOH} / \text { glycid } \\
\text { yl trimethyl } \\
\text { ammonium } \\
\text { chloride } \\
\text { impregnation }\end{array}$ & $\begin{array}{l}\text { Vanadiu } \\
\mathrm{m}\end{array}$ & $\begin{array}{l}\text { Freundlic } \\
\mathrm{h} \text { and } \\
\text { Redlich- } \\
\text { Peterson }\end{array}$ & - & $>90 \%$ & $\begin{array}{l}\mathrm{pH}: 3 \text {, } \\
\text { dosage: } \\
2 \mathrm{~g} / \mathrm{L} ; \mathrm{t}: 24 \mathrm{~h} ; \\
\mathrm{Co}: 20 \mathrm{mg} / \mathrm{L} ; \\
\text { T } 20^{\circ} \mathrm{C}\end{array}$ & $\begin{array}{l}\mathrm{NaCl}(87 \\
\%)\end{array}$ & [56] \\
\hline & $\begin{array}{l}\text { Synthetic } \\
\text { copper } \\
\text { solution } \\
\end{array}$ & $\begin{array}{l}\mathrm{HCl} \\
\text { impregnation }\end{array}$ & $\mathrm{CU}(\mathrm{II})$ & Langmuir & $\begin{array}{l}\text { Pseudo- } \\
\text { second } \\
\text { order }\end{array}$ & $0.071 \mathrm{mmol} / \mathrm{g}$ & $\mathrm{pH} 4-6$ & - & [57] \\
\hline
\end{tabular}




\begin{tabular}{|c|c|c|c|c|c|c|c|c|c|}
\hline Sorbent & $\begin{array}{l}\text { Aqueous } \\
\text { Media }\end{array}$ & $\begin{array}{l}\text { Modification } \\
\text { Technique }\end{array}$ & Pollutant & $\begin{array}{l}\text { Isoterm } \\
\text { Model }\end{array}$ & Kinetics & $\begin{array}{l}\text { Removal } \\
\text { Efficiency } \\
\text { (\%)/ } \\
\text { Adsorption } \\
\text { Capacity } \\
\text { (mg/g) }\end{array}$ & $\begin{array}{l}\text { Optimum } \\
\text { Condition }\end{array}$ & $\begin{array}{l}\text { Desorption } \\
\text { Solution/ } \\
\text { Regenera } \\
\text { tion (\%) }\end{array}$ & Ref. \\
\hline \multirow[t]{3}{*}{$\begin{array}{l}\text { Rice } \\
\text { husk }\end{array}$} & $\begin{array}{l}\text { Synthetic } \\
\text { phenol } \\
\text { solution }\end{array}$ & $\begin{array}{l}\text { Carbonizatio } \\
\text { n } 400^{\circ} \mathrm{C}\end{array}$ & Phenol & - & - & $64.9 \%$ & $\begin{array}{l}\text { Surface area: } \\
201 \mathrm{~m}^{2} / \mathrm{g}, \\
\text { stirring: } 200 \\
\text { rpm, } \\
\text { temperatu- } \\
\text { re: } 23 \pm 1^{\circ} \mathrm{C} \text {. } \\
\text { dosage: } 10 \\
\text { g/L, Co: } 100 \\
\mathrm{mg} / \mathrm{L}, \mathrm{pH}: \\
5.58\end{array}$ & - & [63] \\
\hline & $\begin{array}{l}\text { Synthetic } \\
\text { dyes } \\
\text { solution }\end{array}$ & $\begin{array}{l}\mathrm{HCl} \\
\text { impregnation }\end{array}$ & $\begin{array}{l}\text { Direct } \\
\text { Orange, } \\
\text { Direct } \\
\text { Red } \\
\text { Direct, } \\
\text { Ever } \\
\text { direct } \\
\text { Orange }\end{array}$ & - & $\begin{array}{l}\text { Pseudo- } \\
\text { second } \\
\text { order }\end{array}$ & $20-32 \mathrm{mg} / \mathrm{g}$ & $\begin{array}{l}\text { Stirring } \\
: 100 \mathrm{rpm}, \mathrm{T}: \\
30^{\circ} \mathrm{C}, \mathrm{t}: 180 \\
\text { min }\end{array}$ & $\begin{array}{l}\text { Distilled } \\
\text { water }\end{array}$ & [64] \\
\hline & $\begin{array}{l}\text { Synthetic } \\
\text { phenol } \\
\text { solution }\end{array}$ & $\begin{array}{l}\mathrm{KOH} \\
\text { impregnation } \\
\text { / Pyrolysis } \\
750^{\circ} \mathrm{C}\end{array}$ & Phenol & Langmuir & $\begin{array}{l}\text { Pseudo- } \\
\text { second } \\
\text { order }\end{array}$ & $201 \mathrm{mg} / \mathrm{g}$ & $\begin{array}{l}\text { Surface area: } \\
2138 \mathrm{~m} 2 / \mathrm{g} \\
\mathrm{pH}: 7, \mathrm{C}_{\circ} \\
0.5 \mathrm{~g} / \mathrm{L}, \mathrm{t}: 240 \\
\min \end{array}$ & - & [65] \\
\hline $\begin{array}{l}\text { Platanu } \\
\text { s } \\
\text { orientali } \\
\text { s leaves }\end{array}$ & $\begin{array}{l}\text { Synthetic } \\
\text { dyes } \\
\text { solution }\end{array}$ & $\begin{array}{l}\text { Carbonizatio } \\
\mathrm{n} \\
210^{\circ} \mathrm{C} / \mathrm{Calcin} \\
\text { ation } \\
750^{\circ} \mathrm{C} / \mathrm{KOH} / \\
\mathrm{H}_{2} \mathrm{SO}_{4} \\
\text { impregnation }\end{array}$ & $\begin{array}{l}\text { Rhoda- } \\
\text { min B } \\
\text { Metylene } \\
\text {-blue }\end{array}$ & Langmuir & $\begin{array}{l}\text { Pseudo- } \\
\text { second } \\
\text { order }\end{array}$ & $\begin{array}{l}557.06 \mathrm{mg} / \mathrm{g} \\
327.49 \mathrm{mg} / \mathrm{g}\end{array}$ & - & - & [66] \\
\hline $\begin{array}{l}\text { Orange } \\
\text { peel }\end{array}$ & $\begin{array}{l}\text { Synthetic } \\
\text { methylene } \\
\text { blue dye } \\
\text { solution }\end{array}$ & $\begin{array}{l}\mathrm{ZnCl} \\
\text { activation/ } \\
\text { Carbonizatio } \\
\mathrm{n}\end{array}$ & $\begin{array}{l}\text { Methyle- } \\
\text { ne blue } \\
\text { dye }\end{array}$ & $\begin{array}{l}\text { Langmuir } \\
\text { and Sips }\end{array}$ & $\begin{array}{l}\text { Pseudo- } \\
\text { second } \\
\text { order }\end{array}$ & $99 \%$ & $\begin{array}{l}\mathrm{pH}: 7-8, \mathrm{~T}: \\
25 \pm 2{ }^{\circ} \mathrm{C} \\
\text { Stirring: } 120 \mathrm{rp} \\
\mathrm{m}, \mathrm{t}: 144 \mathrm{~min}\end{array}$ & - & [71] \\
\hline \multirow[t]{3}{*}{$\begin{array}{l}\text { Banana } \\
\text { peel }\end{array}$} & $\begin{array}{l}\text { Synthetic } \\
\text { methylene } \\
\text { blue dye } \\
\text { solution }\end{array}$ & $\begin{array}{l}\mathrm{FeSO} 4 \\
\text { activation- } \\
\text { carbonizatio } \\
\mathrm{n}\end{array}$ & $\begin{array}{l}\text { Methyle- } \\
\text { ne blue } \\
\text { dye }\end{array}$ & Langmuir & $\begin{array}{l}\text { Pseudo } \\
\text { second } \\
\text { order }\end{array}$ & $862 \mathrm{mg} / \mathrm{g}$ & $\begin{array}{l}\mathrm{C}_{0}: 500 \mathrm{mg} / \mathrm{L}, \\
\mathrm{pH}: 6.1 \\
\mathrm{~T}: 313 \mathrm{~K}, \\
\text { stirring: } 180 \\
\text { rpm }\end{array}$ & $\begin{array}{l}\text { Anhydrou } \\
\text { s ethanol, } \\
5 \text { cycles }\end{array}$ & {$[58]$} \\
\hline & $\begin{array}{l}\text { Synthetic } \\
\text { solution } \\
\mathrm{NiCl}_{2} \cdot 6 \mathrm{H}_{2} \mathrm{O}\end{array}$ & $\begin{array}{l}\mathrm{H}_{3} \mathrm{PO}_{4} \\
\text { activation/ } \\
\text { hydrothermal }\end{array}$ & $\mathrm{Ni}(I I)$ & Langmuir & & $320 \mathrm{mg} / \mathrm{g}$ & $\begin{array}{l}\mathrm{pH}: 6, \mathrm{~T}: 500^{\circ} \mathrm{C}, \\
\text { stirring: } 150 \\
\text { rpm }\end{array}$ & & [72] \\
\hline & & $\begin{array}{l}\mathrm{H}_{3} \mathrm{PO}_{4} \\
\text { activation/ } \\
\text { pyrolysis }\end{array}$ & & & & $488 \mathrm{mg} / \mathrm{g}$ & & & \\
\hline \multirow[t]{2}{*}{$\begin{array}{l}\text { Jackfrui } \\
\text { † peel }\end{array}$} & $\begin{array}{l}\text { Synthetic } \\
\text { copper } \\
\text { solution }\end{array}$ & $\begin{array}{l}\text { Carbonizatio } \\
\mathrm{n}\end{array}$ & $\mathrm{Cu}(\mathrm{II})$ & - & - & $99.84 \%$ & $\begin{array}{l}\text { Temperatu- } \\
\text { re: } 45^{\circ} \mathrm{C}, \mathrm{pH}: \\
7, \mathrm{t}: 144 \mathrm{~min}, \\
\mathrm{C}_{\circ}: 100 \mathrm{ppm} \text {, } \\
\text { surface area: } \\
3.6461 \mathrm{~m}^{2} / \mathrm{g}\end{array}$ & - & [73] \\
\hline & $\begin{array}{l}\text { Synthetic } \\
\mathrm{Cr}\left(\mathrm{NO}_{3}\right)_{3} \\
\mathrm{NiSO}_{4} \\
\text { solution }\end{array}$ & $\begin{array}{l}\text { EDTA } \\
\text { activation } \\
\text { SDS } \\
\text { activation } \\
\mathrm{NaOH} \\
\text { activation } \\
\mathrm{HNO}_{3} \\
\text { Raw/ drying }\end{array}$ & $\begin{array}{l}\mathrm{Cr}(I I I) \\
\mathrm{Ni}(I I) \\
\mathrm{Cr}(\mathrm{III}) \\
\mathrm{Ni}(\mathrm{II}) \\
\mathrm{Cr}(\mathrm{III}) \\
\mathrm{Ni}(\mathrm{II}) \\
\mathrm{Cr}(\mathrm{III}) \\
\mathrm{Ni}(\mathrm{II}) \\
\mathrm{Cr}(\mathrm{III}) \\
\mathrm{Ni}(\mathrm{II})\end{array}$ & Langmuir & $\begin{array}{l}\text { Pseudo } \\
\text { second } \\
\text { order }\end{array}$ & $\begin{array}{l}41.67 \mathrm{mg} / \mathrm{g} \\
52.08 \mathrm{mg} / \mathrm{g} \\
26.25 \mathrm{mg} / \mathrm{g} \\
20.88 \mathrm{mg} / \mathrm{g} \\
37.04 \mathrm{mg} / \mathrm{g} \\
27.17 \mathrm{mg} / \mathrm{g} \\
25.25 \mathrm{mg} / \mathrm{g} \\
21.88 \mathrm{mg} / \mathrm{g} \\
13.5 \mathrm{mg} / \mathrm{g} \\
12.03 \mathrm{mg} / \mathrm{g}\end{array}$ & $\begin{array}{l}\text { Time: } 30-60 \\
\text { min, } \mathrm{pH}: 4-6 \text {, } \\
\text { dosage: } 0.1 \\
\mathrm{~g} / \mathrm{L}\end{array}$ & $\begin{array}{l}\mathrm{pH} 1-2 \\
(20 \%-36 \\
\%)\end{array}$ & {$[74]$} \\
\hline
\end{tabular}




\begin{tabular}{|c|c|c|c|c|c|c|c|c|c|}
\hline Sorbent & $\begin{array}{l}\text { Aqueous } \\
\text { Media }\end{array}$ & $\begin{array}{l}\text { Modification } \\
\text { Technique }\end{array}$ & Pollutant & $\begin{array}{l}\text { Isoterm } \\
\text { Model }\end{array}$ & Kinetics & $\begin{array}{l}\text { Removal } \\
\text { Efficiency } \\
\text { (\%)/ } \\
\text { Adsorption } \\
\text { Capacity } \\
\text { (mg/g) }\end{array}$ & $\begin{array}{l}\text { Optimum } \\
\text { Condition }\end{array}$ & $\begin{array}{l}\text { Desorption } \\
\text { Solution/ } \\
\text { Regenera } \\
\text { tion (\%) }\end{array}$ & Ref. \\
\hline $\begin{array}{l}\text { Jackfrui } \\
\text { † seed }\end{array}$ & $\begin{array}{l}\text { Synthetic } \\
\text { malachite } \\
\text { green } \\
\text { oxalate } \\
\text { solution }\end{array}$ & Drying & $\begin{array}{l}\text { Malachit } \\
\text { e green } \\
\text { dye }\end{array}$ & Freudlich & $\begin{array}{l}\text { Pseudo } \\
\text { second } \\
\text { order }\end{array}$ & $66 \mathrm{mg} / \mathrm{g}$ & $\begin{array}{l}\text { Time: } 120 \\
\text { min, stirring: } \\
250 \text { rpm }\end{array}$ & - & {$[75]$} \\
\hline $\begin{array}{l}\text { Pineap } \\
\text { ple } \\
\text { peel }\end{array}$ & $\begin{array}{l}\text { Synthetic } \\
\text { methylene } \\
\text { blue dye } \\
\text { solution }\end{array}$ & $\begin{array}{l}\text { 1-butyl-3- } \\
\text { methylimidaz } \\
\text { olium } \\
\text { chloride }\end{array}$ & $\begin{array}{l}\text { Methyle- } \\
\text { ne blue } \\
\text { dye }\end{array}$ & Langmuir & $\begin{array}{l}\text { Pseudo } \\
\text { second } \\
\text { order }\end{array}$ & 101.94 mg/g & $\mathrm{pH}: 6$ & $\begin{array}{l}0.1 \mathrm{M} / \mathrm{L} \\
\mathrm{HCl}\end{array}$ & [76] \\
\hline $\begin{array}{l}\text { Melon } \\
\text { peel/hu } \\
\text { sk }\end{array}$ & $\begin{array}{l}\text { Synthetic } \\
\text { metals } \\
\text { solution }\end{array}$ & $\begin{array}{l}\text { ZnS } \\
\text { nanoparticle } \\
\text { activation }\end{array}$ & $\begin{array}{l}\mathrm{Pb}(\mathrm{II}) \\
\mathrm{Cd}(\mathrm{II}) \\
\mathrm{Cu}(\mathrm{II}) \\
\mathrm{Ni}(\mathrm{II}) \\
\mathrm{Co}(\mathrm{II})\end{array}$ & Langmuir & $\begin{array}{l}\text { Pseudo } \\
\text { second } \\
\text { order }\end{array}$ & $\begin{array}{l}106.4 \mathrm{mg} / \mathrm{g} \\
69.7 \mathrm{mg} / \mathrm{g} \\
37.7 \mathrm{mg} / \mathrm{g} \\
40.2 \mathrm{mg} / \mathrm{g} \\
32.8 \mathrm{mg} / \mathrm{g}\end{array}$ & $\begin{array}{l}\text { Temperatu- } \\
\text { re: } 30^{\circ} \mathrm{C}, \\
\mathrm{pH}: 5-6, \\
\text { stirring: } 50 \\
\text { rpm }\end{array}$ & - & {$[62]$} \\
\hline $\begin{array}{l}\text { Durian } \\
\text { shell }\end{array}$ & $\begin{array}{l}\text { Synthetic } \\
\text { solution } \mathrm{Zn} \\
\left(\mathrm{NO}_{3}\right)_{2} \cdot 6 \mathrm{H}_{2} \\
\bigcirc\end{array}$ & $\begin{array}{l}\mathrm{HCl} \\
\text { impregnation } \\
\text { / drying }\end{array}$ & $\mathrm{Zn}(\mathrm{II})$ & Langmuir & $\begin{array}{l}\text { Pseudo- } \\
\text { second } \\
\text { order }\end{array}$ & $36.73 \mathrm{mg} / \mathrm{g}$ & $\begin{array}{l}\mathrm{pH}: 8, \\
\text { dosage: } 0.5 \\
\text { g/L, time: } 4 \mathrm{~h} \text {, } \\
\text { temperatu- } \\
\text { re: } 313 \mathrm{~K} \text {, } \\
\text { stirring } 120 \\
\text { rpm }\end{array}$ & $\begin{array}{l}0.1 \mathrm{M} \mathrm{HCl} \\
(98.9 \%)\end{array}$ & [77] \\
\hline $\begin{array}{l}\text { Fungal } \\
\text { Aspergil } \\
\text { lus } \\
\text { fumigat } \\
\text { us+ } \\
\text { aspergil } \\
\text { lus niger }\end{array}$ & $\begin{array}{l}\text { Synthetic } \\
\mathrm{K}_{2} \mathrm{Cr}_{2} \mathrm{O}_{7} \\
\text { solution }\end{array}$ & $\begin{array}{l}\text { Incubation/ } \\
\text { drying, } \\
\text { magnetiza- } \\
\text { tion }\end{array}$ & $\mathrm{Cr}(\mathrm{VI})$ & $\begin{array}{l}\text { Freundlic } \\
\mathrm{h}\end{array}$ & $\begin{array}{l}\text { Pseudo-first } \\
\text { order }\end{array}$ & $249.9 \mathrm{mg} / \mathrm{g}$ & $\begin{array}{l}\mathrm{C}_{\circ}: 50 \mathrm{mg} / \mathrm{L}, \\
\mathrm{pH}: 2, \\
\text { dosage: } 0.7 \\
\text { g/L, } \\
\text { temperature: } \\
30 \text { ॰C, time: } \\
60 \text { min, } \\
\text { stirring: } 120 \\
\text { rpm }\end{array}$ & & {$[2]$} \\
\hline
\end{tabular}

\subsection{ANIMAL WASTE BASED-SORBENT}

The increasing number of global population has led to the increasing amount of meat consumption and production. This has caused more animal waste generated from cattle farm and food related industries that has reached millions of tons annually. The disposal needs to be utilized to save the environment and, at the same time, support circular economy system by recycling waste materials. One great loophole to gain these purposes is by valorizing the animal waste to be used as environmentally friendly sorbent [35-37]. Literature shows that animal bone is effective as sorbent material as it contains carbon and other favorable component for binding pollutants such as apatite calcium phosphate having ionic exchange features to attract scattered pollutants [78] (Table 4).

\subsection{Chicken Bone}

To date, the valorization of chicken bone as natural sorbent has covered a wide range of pollutant types and activation modes. To start with, biochar developed from chicken bone treated at lower carbonization temperature showed its polar functional group beneficial to adsorb metal $\mathrm{Cr}(\mathrm{VI})$ ion
[40]. It was further examined to adsorb metal lead ions by conducting pyrolysis on different chicken bone parts [79]. After conducting comparative analysis, this work resumed that chicken tibia bonebased biochar outperformed pelvat, ribs, clavicle, vertebrae, and humerus bone-based biochars. Later, it informed that metal ions trapped on the sorbent surface were mostly able to desorb using acid solution confirming its considerable regeneration rate. The next study created a novel chicken bone with double coating using iron and magnesium hydroxides and proclaimed that cadmium metal ions contained in solution could be mostly reduced [80]. Regarding dyes eradication, dye pollutant named basic fuchsine could also be removed from aqueous media using activated carbon and biochar derived from chicken bone in separate experiments. This study claimed that activated carbon was more dependable than biochar [81].

\subsection{Fish Bone}

As part of abundant animal wastes, the utilization of fish bone is worthy to do as it has competitive efficacy with other organic materials. A study proved that the performance of bone char from pleco fish (Pterygoplichthys spp.) was promising for both fluoride and $\mathrm{Cd}(I I)$ metal ion removal [82]. With surface area 
around $110 \mathrm{~m}^{2} / \mathrm{g}$, it worked better at lower $\mathrm{pH}$ for fluoride and higher $\mathrm{pH}$ for $\mathrm{Cd}(\mathrm{II})$ removal. Additionally, a deeper characterization analysis indicated that electrostatic attractions, ion exchange, and physisorption happened during the adsorption process.

The implementation of this sorbent in treating synthetic and raw aqueous media may have some differences due to the complexity of the solution in nature. Raw wastewater from abattoirs, for instance, is one of those complex mixtures. Regarding this issue, a study utilized fish bone chito-protein as both sorbent and coagulant agent for reducing particle load [83]. It found expectant result as most of the particle load could be reduced as well as BOD concentration.

Thus, the bone synthesis of other fish species namely Catla fish was also informed reliable by successfully removing around $77 \%$ of crystal violet and $87 \%$ congo red dyes within 75 min along with the addition of photo catalytic treatment [78]. Other work then studied to carbonize bone waste derived from tilapia fish bone, and it reported credible outcome of tetracycline removal from aqueous solution [84]. Based on the experimental work, it could be assumed that, at near neutral $\mathrm{pH}$, tetracycline could present anionic characteristics when the sorbent surface was positively charged leading to better adsorption capacity.

\subsection{Bovine Bone}

The usage of bovine bone-based sorbent has been notified in related literature. To begin with, bovine bone-based sorbent that was initially activated using $\mathrm{NaOH}$ impregnation was reported successful to separate chromium ions from raw chrome electroplating wastewater [85]. Further, a study harnessed activated carbon derived from this bone. By having excellent surface area $3231.8 \mathrm{~m}^{2} /$, it confirmed a great adsorption capacity of the sorbent regarding the eradication of antibiotics pollutants namely sulfamethazine and chloramphenicol by around $1194 \mathrm{mg} / \mathrm{g}$ and $1240 \mathrm{mg} / \mathrm{g}$, respectively [39]. Thus the brushite sorbent derived from bovine bone which was used to separate $\mathrm{Cr}$ (III) metal ions from aqueous solutions could reach up to $95 \%$ of pollutant removal [86]. All those reports agreed with the potentiality of bovine bone as an alternative option for greener sorbent. Eventually, a further study conducted a comparative investigation of various bovine bone parts such as ribs, scapulae, vertebrae, and legs by individually testing their competence under the same operating condition. Bovine rib bonebased biochar then was informed to have the highest sorption capacity followed by scapulae, vertebrae, and legs bone-based sorbents [87].

\subsection{Cow Bone}

Cow farm can be considered as the most important part of meat industry globally resulting in massive cow bone waste. Therefore, cow bone availability is quite sufficient for producing organic sorbent. A study then made nitrogenous biochar from the bone by implementing a ball-milling technique, and it informed that this breakthrough was effective by reaching satisfactory heavy metals reduction [42]. Moreover, other study revealed that cow bonebased sorbent could be an ideal option for purifying raw cassava wastewater as it could obtain high adsorption capacity for several heavy metals removal using at least $0.02 \mathrm{~g}$ of this sorbent dosage [88]. Compared to any available commercial sorbents, the outcome aforementioned was highly competitive.

\subsection{Ostrich Bone}

Ostrich bone-based sorbent was also confirmed excellent to capture pollutants. Heavy metal lead ions, for example, could be taken out of aqueous solution only within 5 min of contact time [89]. Other research then improved the nature of ostrich bone as novel composite ash/nanoscale zerovalent iron for adsorbing both $\mathrm{Hg}(\mathrm{II})$ and $\mathrm{Pb}(\mathrm{II})$ metal ions in a fixedbed column mode [90]. However, the efficacy of this modified ostrich bone was not equal to all types of metal ions as $\mathrm{Hg}(\mathrm{II})$ and $\mathrm{Pb}(\mathrm{II})$ have higher removal efficiency than that of $\mathrm{Ni}(\mathrm{II})$ and $\mathrm{Cd}(\mathrm{II})$. In accordance with its reusability, both fresh and used sorbent were regarded economical due to relative stability of sorbent performance even after multiple adsorption stages [91].

\subsection{Camel Bone}

Camel population, especially in Mediterranean countries, has increased due to an increasing demand of meat industry, tourism, and traditional transportation. The escalating amount of camel carcasses then could be another issue. Fortunately, utilizing them as sorbent material could be part of green solution for environment. Some research has reported the competence of this low cost material to adsorb various pollutants especially heavy metals ions. Camel bone-based sorbent for $\mathrm{Cd}(\mathrm{II})$ and $\mathrm{Pb}(\mathrm{II})$ ions removal, for instance, was notified well [92]. By applying different pyrolysis temperatures of 500, 600, 800 , and $900^{\circ} \mathrm{C}$, the study found $800^{\circ} \mathrm{C}$ as the optimum pyrolysis level resulting in $\mathrm{Cd}(\mathrm{II})$ and $\mathrm{Pb}(\mathrm{II})$ removal by around $99.4 \%$ and $99.89 \%$, respectively. Other research then produced magnetic composite sorbent extracted from camel bones by involving chemical coating $\mathrm{Fe}_{3} \mathrm{O}_{4}$ on sorbent surface [93]. It found the existence of chemical elements like $\mathrm{Ca}, \mathrm{P}$, $C$ on the modified sorbent that influenced its sorption capacity which reached $315 \mathrm{mg} / \mathrm{g}, 300.8 \mathrm{mg} / \mathrm{g}$, and $271.7 \mathrm{mg} / \mathrm{g}$ for $\mathrm{Pb}(\mathrm{II}), \mathrm{Cd}(\mathrm{II})$, and $\mathrm{Co}(\mathrm{II})$, respectively. Further, camel bone activated by both sodium hydroxide impregnation and carbonization was reported considerable to eliminate $\mathrm{CU}(\mathrm{II})$ metal ions from solution in industrial semi-pilot column mode [94]. 


\subsection{Pig Bone}

The utilization of pig bone as another sorbent material is interesting to be explored. Literature showed that pig bone-based sorbent initially modified via calcination and mineralization was effective for adsorbing $\mathrm{Pb}(\mathrm{II})$ metal ions [95]. The activation techniques could increase the adsorption capacity from $96.1 \mathrm{mg} / \mathrm{g}$ to $312.5 \mathrm{mg} / \mathrm{g}$. Forward, the development of hydroxyapatite nanocrystal sorbent derived from pig bone to capture hazardous radioisotope pollutant was also successfully conducted [96]. This sorbent has negative charged surface through $\mathrm{NaHCO}_{3}$ chemical impregnation leading to better sorption capacity. In this case, the presence of $\mathrm{CO}_{3}{ }^{2-}$ sites could have a critical function to increase sorbent performance for adsorbing various metal ions including $\mathrm{Cd}(\mathrm{II}), \mathrm{Pb}$ (II), and $\mathrm{CU}(\mathrm{II})$.

\subsection{Other Animal Waste}

Apart from the bones aforementioned, animal wastes also consist of other body parts such as feathers or fur, beaks, hooves, or excretion like the dungs. The increasing interest in exploring the potential of those wastes has contributed to the increasing number of studies investigating various animal remains for sorbent production. To start with, harmful $\mathrm{Cr}(\mathrm{VI})$ metal ions has been successfully removed from solutions by exploiting chicken feathers [97]. At extremely acidic $\mathrm{pH}$, this sorbent could have great monolayer capacity. When the feathers were acidified using nitric acid, the adsorption capacity of $\mathrm{Cr}(\mathrm{VI})$ ions in fixed bed mode was reported to be more satisfactory [98]. However, this report found that other types of metal such as $\mathrm{CU}$ (II), $\mathrm{Mn}$ (II) and Co(II) ions could not be effectively adsorbed. This phenomenon needs to be further analyzed as it could be regarded to the particular metal characteristics behaving different against sorbent characters in nature.

Furthermore, deflouridation process can be done using chemically activated chicken feathers in the batch mode [99]. The experimental data indicated the double increase in fluoride removal when sorbent dosage was increased eightfold. Other study conducted the comparative development of sorbent derived from chicken femur, chicken beak, and fishbone wastes through carbonization temperature of $900^{\circ} \mathrm{C}$ to adsorb $\mathrm{Cd}(\mathrm{II})$ metal ions from raw shipbuilding wastewater [100]. It reported that sorbent derived from chicken femur outperformed chicken beak and fishbone-based sorbents. Additionally, different from previous works that reported acidic $\mathrm{pH}$ favorable, near neutral $\mathrm{pH} 6$ was found optimum to eliminate targeted metal ions from the solution used in this study.

The fabrication of sorbent made from chondroitin extraction waste existed in animal bone was developed to adsorb $\mathrm{Pb}$ (II) metal ions[101]. It revealed that metal removal was leveraged by initial $\mathrm{pH}$ where $\mathrm{pH} 3$ was observed as the best one. Eventually, there have been some other studies reported the usage of dried animal dung for creating alternative sorbent. Cow dung based activated carbon, for example, was proven effective by showing large active surface area leading to satisfactory removal percentage [102]. Another study assessed the performance of bivalve mollusk-based sorbent which was previously given pyrolysis treatment for the removal of rifampicin antibiotic from water [103]. Based on the characterization analysis, residual $\mathrm{CaCO}_{3}$ in the form of aragonite and calcite along with its porous structure on heterogeneous sorbent surface can be linked to the sufficient sorption capacity of the sorbent which could reach of $7 \mathrm{mg} / \mathrm{g}$.

In accordance with comparative analysis between plant and animal wastes, a study informed an interesting analysis dealing with hydrothermal liquefaction process to separately activate rice husk and cow dung under the same operating conditions for removing dye. It reported that remaining congo red dye in raw textile wastewater could be lowered up to $96.9 \%$ and $98.8 \%$ using rice husk and cow dung based- sorbents, respectively [104]. From this point of view, it can be firmly declared that both plant and animal waste are comparatively reliable to other natural and commercial sorbents for adsorbing various pollutants from diverse aqueous media.

Table 4 The application of natural sorbents derived from animal wastes

\begin{tabular}{|c|c|c|c|c|c|c|c|c|c|}
\hline Sorbent & $\begin{array}{l}\text { Aqueous } \\
\text { Media }\end{array}$ & $\begin{array}{l}\text { Activation } \\
\text { Technique }\end{array}$ & $\begin{array}{l}\text { Targeted } \\
\text { Pollutant }\end{array}$ & $\begin{array}{l}\text { Isoterm } \\
\text { Model }\end{array}$ & Kinetics & $\begin{array}{l}\text { Removal } \\
\text { Efficiency (\%)/ } \\
\text { Adsorption } \\
\text { Capacity }(\mathrm{mg} / \mathrm{g})\end{array}$ & $\begin{array}{l}\text { Optimum } \\
\text { Condition }\end{array}$ & $\begin{array}{l}\text { Desorption } \\
\text { Solution/ } \\
\text { Regenera- } \\
\text { tion (\%) }\end{array}$ & Ref. \\
\hline \multirow[t]{2}{*}{$\begin{array}{l}\text { Chicken } \\
\text { bone }\end{array}$} & $\begin{array}{l}\text { Synthetic } \\
\mathrm{K}_{2} \mathrm{Cr}_{2} \mathrm{O}_{7} \\
\text { solution }\end{array}$ & $\begin{array}{l}\text { Pyrolysis } \\
350^{\circ} \mathrm{C}\end{array}$ & $\mathrm{Cr}(\mathrm{VI})$ & Langmuir & Elovich & $58.195 \mathrm{mg} / \mathrm{g}$ & $\begin{array}{l}\mathrm{pH}: 2, \\
\text { stirring:150 } \\
\text { rpm }\end{array}$ & $\begin{array}{l}0.1 \mathrm{NaOH} \\
(90 \%)\end{array}$ & {$[40]$} \\
\hline & $\begin{array}{l}\text { Synthetic } \\
\mathrm{Pb}\left(\mathrm{NO}_{3}\right)_{2} \\
\text { solution }\end{array}$ & $\begin{array}{l}\text { Pyrolysis } \\
600^{\circ} \mathrm{C}\end{array}$ & $\mathrm{Pb}$ & Langmuir & - & $263 \mathrm{mg} / \mathrm{g}$ & $\begin{array}{l}\mathrm{pH}: 5, \mathrm{t}: 144 \\
\min \end{array}$ & $\begin{array}{l}0.1 \mathrm{HCl} \\
(75 \%-88 \\
\%)\end{array}$ & [79] \\
\hline
\end{tabular}




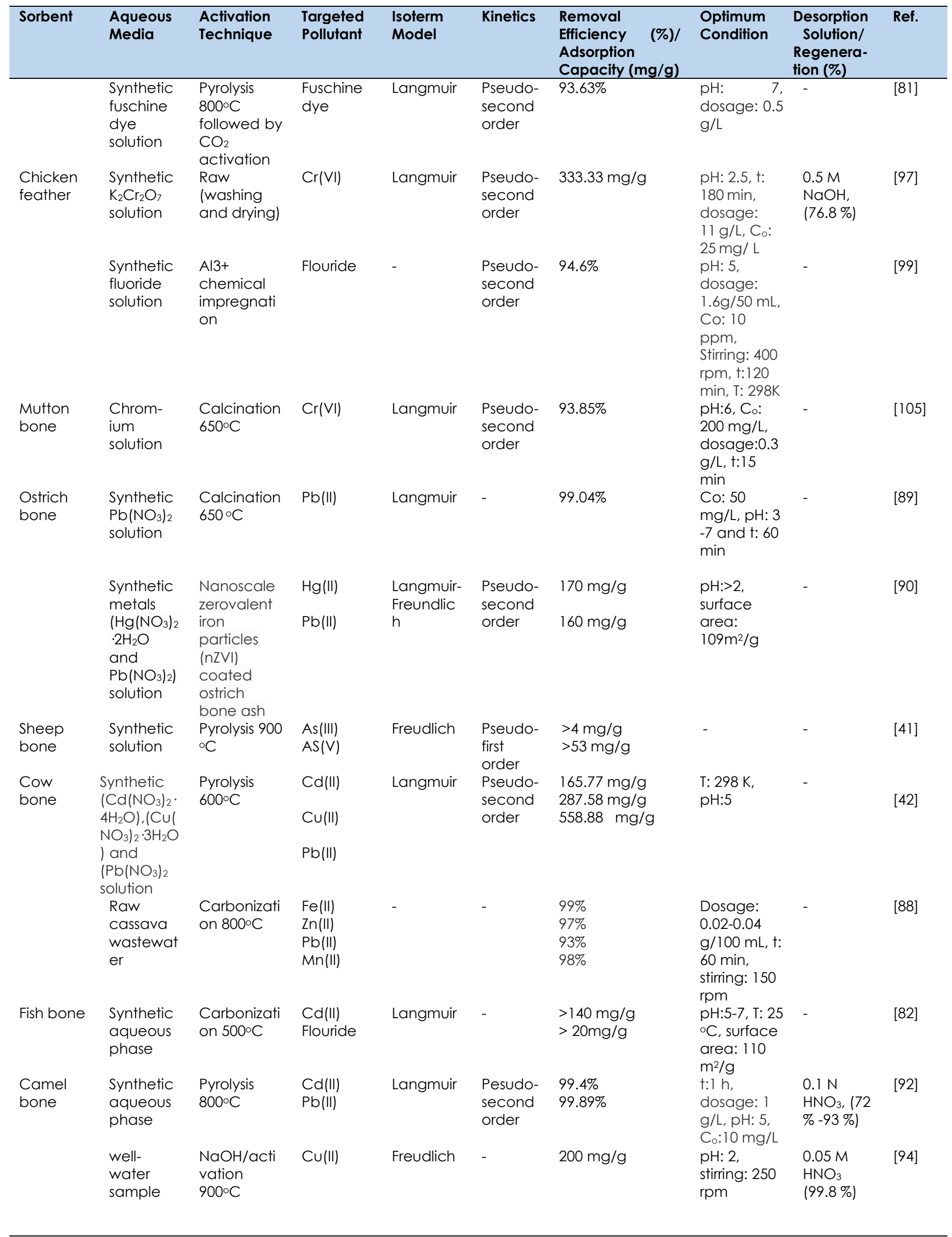




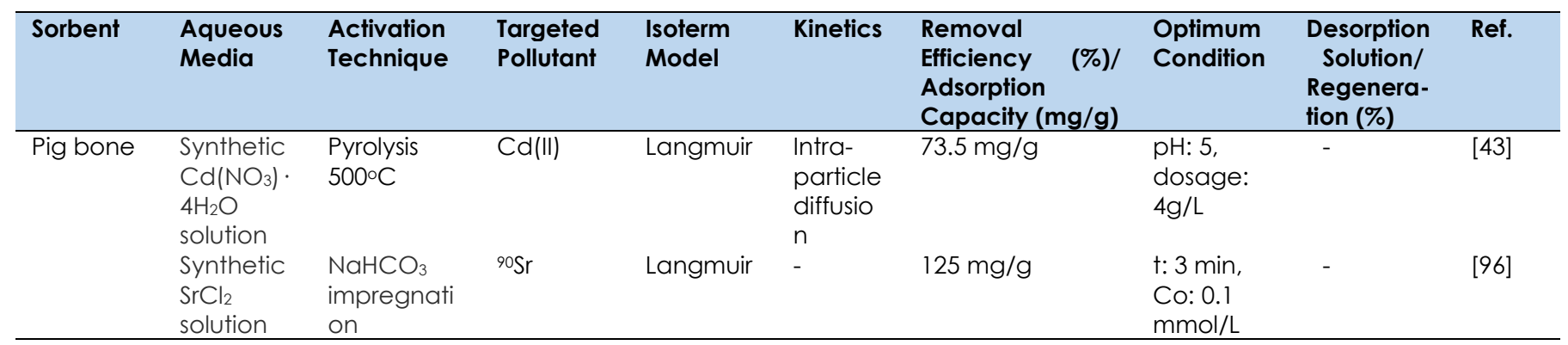

\subsection{CONCLUSIONS, CHALLENGES, AND FUTURE OUTLOOK}

\subsection{Conclusions}

The increasing development of emerging industries and population globally has direct and indirect effect on the increasing amount of polluted aqueous media. This issue must be addressed properly in order to prevent further adverse impact on human health and environment. Therefore, the use of green technology should be prioritized for managing wastewater stream and solid waste in the first stage. Several water and wastewater treatment technologies that can be implemented for pollutants removal including chemical oxidation, biological techniques, and membrane separation are also explained as available option for conducting an integrated adsorption system.

A review on current utilization of organic solid waste namely plant and animal remains as sorbent materials to treat polluted aqueous media then has been presented in this work. Relevant reports in the literature have been comparatively discussed regarding the characteristics and performance of the sorbents.

Furthermore, plant waste like plant bark, plant leaves, rice husk, banana and watermelon peels as well as animal wastes like their bone and fur have been reported reliable as sorbent to adsorb various pollutants contained in synthetic and raw wastewaters. The different modification techniques such as chemical impregnation, physical and thermal activation process for enhancing active surface area and functional group on sorbent surface were also confirmed effective. In accordance with the comparative analysis between plant and animal based sorbents, it can be assumed that using animalbased sorbent tends to give slightly higher outcomes especially for heavy metals reduction. However, it needs further research for other pollutant types. In terms of the availability, plant-based sorbent may be easier to be collected and processed. However, it should be kept in mind that there is no absolute conclusion that one is superior to another as the adsorption capacity and sorbent performance are influenced by multiple factors such as characteristics of synthetic and raw aqueous media, characters and concentration of targeted pollutants, modification procedures given to the raw sorbent, operating conditions, species of those animals or plants, and part of biomass used as sorbent like bone, feather, nail, or dung for animal-based sorbent, and leaves, bark, peel, crown, or seed for plant-based sorbent.

To conclude, it is predicted that the exploration and utilization of biomass as sorbent material will be increasing in the near future due to the increasing awareness of keeping the quality of environment and the interest of modern society in applying circular economy system.

\subsection{Challenges and Future Outlook}

To date, relevant research articles regarding the usage of plant and animal waste-based sorbent to remove various pollutants mostly put their concern to some aspects related to the technicality and performance aspects. Despite modifications or initial preparation techniques showing their function to boost sorbent performance, more discussions related to the analysis of cost effectiveness have to be considered, especially when we talk about its effectiveness and efficiency for being applied for upgrading into wider scale application in real industrial wastewater treatment plant. It is also worth to compare them with the available commercial sorbents derived from other precursors. Other than that, several unpredictable outcomes between the experimental and theoretical findings referred to sorbent capacity and operating conditions or activation modes still can be found in less ideal condition of sorbent surface for upgrading purposes. The next concern is finding low cost and environmentally friendly regeneration process to obtain organic sorbent with great durability and sustainable disposal.

Furthermore, to narrow other gaps related to modification procedures and species diversity, more initial activation pathways both on the conventional and novel biomass may be worth to be investigated to show the difference of the breakthrough. It then could lead to better comparative analysis among those modification techniques, operating conditions, and the changes of sorbent properties. The need of employing both synthetic and raw wastewaters containing targeted pollutants should be strongly encouraged to get comprehensive perspective related to the performance of modified sorbent in the real solutions as this precinct would bring more 
dependable analysis. Eventually, as the existing literature mostly targeted the prominent unwanted particles such as heavy metals, dyes, and oil compounds, future research could be more focus on applying those biomass for removing other types of pollutants from aqueous media especially those which have not been widely investigated due to its small percentage in the aqueous media composition.

\section{Acknowledgement}

The authors thank Universitas Muhammadiyah Palembang and Curtin University for support given during finalizing this work. There is no conflict of interest of this work.

\section{References}

[1] Mishra, S., Cheng, L. and Maiti, A. 2020. The Utilization of Agro-biomass/byproducts for Effective Bio-removal of Dyes from Dyeing Wastewater: A Comprehensive Review. Journal of Environmental Chemical Engineering. 9(1): 104901.

DOI: https://doi.org/10.1016/j.jece.2020.104901.

[2] Saravanan, A., Kumar, P. S., Govarthanan, M., George, C. S., Vaishnavi, S., Moulishwaran, B. \& Kumar, S. P. 2021. Adsorption Characteristics of Magnetic Nanoparticles Coated Mixed Fungal Biomass for Toxic $\mathrm{Cr}$ (VI) lons in Aquatic Environment. Chemosphere. 267: 129226. DOI: https://doi.org/10.1016/j.chemosphere.2020.129226.

[3] Karimi-Maleh, H., Shafieizadeh, M., Taher, M. A., Opoku, F., Kiarii, E. M., Govender, P. P., Ranjbari, S., Rezapour, M. \& Orooji, Y. 2020. The Role of Magnetite/graphene Oxide Nano-composite as a High-efficiency Adsorbent for Removal of Phenazopyridine Residues from Water Samples, an Experimental/theoretical Investigation. Journal of Molecular Liquids. 298: 112040.

DOI: https://doi.org/10.1016/j.chemosphere.2020.129226.

[4] Esmaeili, H., Tamjidi, S., and Abed, M. 2020. Removal of Cu (II), $\mathrm{Co}$ (II) and Pb (II) from Synthetic and Real Wastewater using Calcified Solamen Vaillanti Snail Shell. Desalination and Water Treatment. 174: 324-335.

DOI: https://doi.org/10.5004/dwt.2020.24880.

[5] Chakraborty, R., Asthana, A., Singh, A. K., Jain, B. \& Susan, A. B. H. 2020. Adsorption of Heavy Metal lons by Various Low-cost Adsorbents: A Review. International Journal of Environmental Analytical Chemistry. 1-38.

DOI: https://doi.org/10.1080/03067319.2020.172281.

[6] Methneni, N., Morales González, J. A., Jaziri, A., Ben Mansour, H. \& Fernandez-Serrano, M. 2021. Persistent Organic and Inorganic Pollutants in the Effluents from the Textile Dyeing Industries: Ecotoxicology Appraisal via a Battery of Biotests. Environmental Research. 196: 110956. DOI: https://doi.org/10.1016/j.envres.2021.110956.

[7] Guo, Y., Xue, Q., Zhang, H., Wang, N., Chang, S., Wang, H. Pang, H. \& Chen, H. 2018. Treatment of Real Benzene Dye Intermediates Wastewater by the Fenton Method: Characteristics and Multi-response Optimization. RSC Advances. 8(1): 80-90.

DOI: https://doi.org/10.1039/C7RA09404C.

[8] Kusworo, T. D., Kumoro, A. C., and Utomo, D. P. 2021. Phenol and Ammonia Removal in Petroleum Refinery Wastewater using a Poly(Vinyl) Alcohol Coated Polysulfone Nanohybrid Membrane. Journal of Water Process Engineering. 39: 101718.

DOI: https://doi.org/10.1016/j.jwpe.2020.101718.
[9] Treviño-Reséndez, J. d. J., Medel, A., and Meas, Y. 2021. Electrochemical Technologies for Treating Petroleum Industry Wastewater. Current Opinion in Electrochemistry. 27: 100690

DOI: https://doi.org/10.1016/j.coelec.2021.100690.

[10] Martini, S., Afroze, S., \& Ahmad Roni, K. 2020. Modified Eucalyptus Bark as a Sorbent for Simultaneous Removal of COD, Oil, and $\mathrm{Cr}(\mathrm{III})$ from Industrial Wastewater. Alexandria Engineering Journal. 59(3): 1637-1648. DOI: https://doi.org/10.1016/j.aej.2020.04.010.

[11] Martini, S. and Ang, H. M. 2019. Hybrid TiO2/UV/PVDF Ultrafiltration Membrane for Raw Canola Oil Wastewater Treatment. Desalination and Water Treatment. 148: 51-59. DOI: https://doi.org/10.5004/dwt.2019.23771.

[12] Martini, S., Ang, H. M. \& Znad, H. 2017. Integrated Ultrafiltration Membrane Unit for Efficient Petroleum Refinery Effluent Treatment. CLEAN - Soil, Air, Water. 45: 1600342.

DOI: https://doi.org/10.1002/clen.201600342.

[13] Willner, J. and Fornalczyk, A. 2020. Application of Biological Method for Removing Selected Heavy Metals from Sewage Sludge. Physicochemical Problems of Mineral Processing. 56(2): 387-395. DOI: https://doi.org/10.3790/ppmp/1 18253.

[14] Tamjidi, S. and Esmaeili, H. 2019. Chemically Modified $\mathrm{CaO} / \mathrm{Fe} 3 \mathrm{O} 4$ Nanocomposite by Sodium Dodecyl Sulfate for $\mathrm{Cr}$ (III) Removal from Water. Chemical Engineering \& Technology. 42(3): 607-616. DOI: https://doi.org/10.1002/ceat.201800488

[15] Feng, J., Zhang, J., Song, W., Liu, J., Hu, Z. \& Bao, B. 2020. An Environmental-friendly Magnetic Bio-adsorbent for High-efficiency $\mathrm{Pb}$ ( II ) Removal: Preparation, Characterization and Its Adsorption Performance. Ecotoxicology and Environmental Safety. 203: 111002. DOI: https://doi.org/10.1016/j.ecoenv.2020.111002.

[16] Arora, R. 2021. Nano Adsorbents for Removing the Arsenic from Waste/Ground Water for Energy and Environment Management-A Review. Materials Today: Proceedings. 45(6): 4437-4440.

DOl: https://doi.org/10.1016/j.matpr.2020.12.546.

[17] Agarwal, A., Upadhyay, U., Sreedhar, I., Singh, S. A. \& Patel, C. M. 2020. A Review on Valorization of Biomass in Heavy Metal Removal from Wastewater. Journal of Water Process Engineering. 38: 101602. DOl: https://doi.org/10.1016/j.jwpe.2020.101602.

[18] Ghaedi, A. M. and Vafaei, A. 2017. Applications of Artificial Neural Networks for Adsorption Removal of Dyes from Aqueous Solution: A Review. Advances in Colloid and Interface Science. 245: 20-39. DOI: https://doi.org/10.1016/j.cis.2017.04.015

[19] Martini, S. and Roni, K. A. 2021. The Existing Technology and the Application of Digital Artificial Intelligent in the Wastewater Treatment Area: A Review Paper. Journal of Physics: Conference Series. 1858: 012013. DOl: https://doi:10.1088/1742-6596/1858/1/012013.

[20] Yang, C., Xu, W., Nan, Y., Wang, Y., Hu, Y., Gao, C. \& Chen, X. 2020. Fabrication and Characterization of a High Performance Polyimide Ultrafiltration Membrane for Dye Removal. Journal of Colloid and Interface Science. 562: 589-597. DOI: https://doi.org/10.1016/j.jcis.2019.11.075.

[21] Liu, X., Jiang, B., Yin, X., Ma, H., \& Hsiao, B. S. 2020. Highly Permeable Nanofibrous Composite Microfiltration Membranes for Removal of Nanoparticles and Heavy Metal Ions. Separation and Purification Technology. 233: 115976. DOI: https://doi.org/10.1016/j.seppur.2019.115976.

[22] Lv, J., Zhang, G., Zhang, H., Zhao, C. \& Yang, F. 2018. Improvement of Antifouling Performances for Modified PVDF Ultrafiltration Membrane with Hydrophilic Cellulose Nanocrystal. Applied Surface Science. 440: 1091-1100. DOI: https://doi.org/10.1016/j.apsusc.2018.01.256.

[23] Al Aani, S., Mustafa, T.N. and Hilal. N. 2020. Ultrafiltration Membranes for Wastewater and Water Process 
Engineering: A Comprehensive Statistical Review over the Past Decade. Journal of Water Process Engineering. 35: 101241.

DOI: https://doi.org/10.1016/j.jwpe.2020.101241.

[24] Khan, I. A., Lee, Y. S. and Kim, J. O. 2020. A Comparison of Variations in Blocking Mechanisms of Membrane-fouling Models for Estimating Flux During Water Treatment. Chemosphere. 259: 127328.

Doi: https://doi.org/10.1016/j.chemosphere.2020.127328.

[25] Aljuboury, D.a.d.A. and Shaik, F. 2021. Assessment of $\mathrm{TiO} / \mathrm{ZnO} / \mathrm{H} 2 \mathrm{O} 2$ Photocatalyst to Treat Wastewater from Oil Refinery within Visible Light Circumstances. South African Journal of Chemical Engineering. 35: 69-77. DOI: https://doi.org/10.1016/j.sajce.2020.11.004.

[26] Garrido-Cardenas, J. A., Esteban-García, B., Agüera, A., Sánchez-Pérez, J. A., \& Manzano-Agugliaro, F. 2020. Wastewater Treatment by Advanced Oxidation Process and Their Worldwide Research Trends. International Journal of Environmental Research and Public Health. 17(1): 170. DOI: https://doi.org/10.3390/ijerph17010170.

[27] Tariq, M., Muhammad, M., Khan, J., Raziq, A., Uddin, M. K., Niaz, A., Ahmed, S. S. \& Rahim, A. 2020. Removal of Rhodamine B Dye from Aqueous Solutions using PhotoFenton Processes and Novel Ni-Cu@ MWCNTs Photocatalyst. Journal of Molecular Liquids. 312: 113399. DOI: https://doi.org/10.1016/j.molliq.2020.113399.

[28] Jamil, A., Bokhari, T. H., Javed, T., Mustafa, R., Sajid, M., Noreen, S., Zuber, M., Nazir, A., lqbal, M. \& Jilani, M. I. 2020. Photocatalytic Degradation of Disperse Dye Violet-26 using $\mathrm{TiO} 2$ and $\mathrm{ZnO}$ Nanomaterials and Process Variable Optimization. Journal of Materials Research and Technology. 9(1): 1119-1128. DOI: https://doi.org/10.1016/j.jmrt.2019.11.035.

[29] Mishra, S. and Maiti, A. 2020. Biological Methodologies for Treatment of Textile Wastewater. Environmental Processes and Management. Springer. 77-107. DOI: https://doi.org/10.1007/978-3-030-38152-3.

[30] Gómez-Ramírez, M. and Tenorio-Sánchez, S. A. Treatment of Solid Waste Containing Metals by Biological Methods. Natural Resources Management and Biological Sciences, IntechOpen.

DOI: https://doi.org/10.5772/intechopen.82948.

[31] Venegas, M., Leiva, A. M., Reyes-Contreras, C., Neumann, P., Piña, B. \& Vidal, G. 2021. Presence and Fate of Micropollutants during Anaerobic Digestion of Sewage and Their Implications for the Circular Economy: A Short Review. Journal of Environmental Chemical Engineering. 9(1): 104931. DOI: https://doi.org/10.1016/j.jece.2020.104931.

[32] XU, H., Yang, ,B., Liu, Y., Li, F., Song, X., Cao, X. \& Sand, W. 2021. Evolution of Microbial Populations and Impacts of Microbial Activity in the Anaerobic-Oxic-Settling-Anaerobic Process for Simultaneous Sludge Reduction and Dyeing Wastewater Treatment. Journal of Cleaner Production. 282: 124403 DOI: https://doi.org/10.1016/j.jclepro.2020.124403.

[33] Escudero, L. B. Quintas, P. Y., Wuilloud, R. G. \& Dotto, G. L. 2019. Recent Advances on Elemental Biosorption. Environmental Chemistry Letters. 17(1): 409-427. DOI: https://doi.org/10.1007/s10311-018-0816-6.

[34] R. Bushra, S. Mohamad, Y. Alias, Y. Jin, M. Ahmad. 2021. Current Approaches and Methodologies to Explore the Perceptive Adsorption Mechanism of Dyes on Low-cost Agricultural Waste: A Review, Microporous and Mesoporous Materials. 319 (2021): 111040

[35] Prakash, M. O., Raghavendra, G., Ojha, S. \& Panchal, M. 2020. Characterization of Porous Activated Carbon Prepared from Arhar Stalks by Single Step Chemical Activation Method. Materials Today: Proceedings. 39(4): 1476-1481. DOI: https://doi.org/10.1016/j.matpr.2020.05.370.

[36] Tang, Y., Lin, T., Jiang, C., Zhao, Y. \& Ai, S. 2021. Renewable Adsorbents from Carboxylate-modified Agro-forestry residues for efficient removal of methylene blue dye. Journal of Physics and Chemistry of Solids. 149: 109811. DOI: https://doi.org/10.1016/j.jpcs.2020.109811

[37] Karimi, H., Heidari, M.A., Emrooz, H. B. M. \& Shokouhimehr, M. 2020. Carbonization temperature effects on adsorption performance of metal-organic framework derived nanoporous carbon for removal of methylene blue from wastewater; experimental and spectrometry study. Diamond and Related Materials. 108 (2020) 107999. DOI: https://doi.org/10.1016/j.diamond.2020.107999

[38] Nasir, A.M., Md Nordin, N.A.H., Goh, P.S. \& Ismail, A.F. 2018. Application of two-dimensional leaf-shaped zeolitic imidazolate framework (2D ZIF-L) as arsenite adsorbent: Kinetic, isotherm and mechanism. Journal of Molecular Liquids. 250: 269-277. DOl: https://doi.org/10.1016/j.molliq.2017.12.005

[39] Dai, J. Qin, L. Zhang, R. Xie, A. Chang, Z. Tian, S. Li, C. \& Yan, Y. 2018. Sustainable bovine bone-derived hierarchically porous carbons with excellent adsorption of antibiotics: Equilibrium, kinetic and thermodynamic investigation. Powder Technology. 331: 162-170. DOI: https://doi.org/10.1016/j.powtec.2018.03.005

[40] Yang, T., Han, C., Tang, J., \& Luo, Y. 2020. Removal performance and mechanisms of $\mathrm{Cr}$ (VI) by an in-situ selfimprovement of mesoporous biochar derived from chicken bone. Environmental Science and Pollution Research. 27: 5018-5029.

DOI: https://doi.org/10.1007/s1 1356-019-07116-4

[41] Alkurdi, S.S.A., Al-Juboori, R.A., Bundschuh, J., Bowtell, L. \& McKnight, S. 2020. Effect of pyrolysis conditions on bone char characterization and its ability for arsenic and fluoride removal. Environmental Pollution. 262: 114221. DOI: https://doi.org/10.1063/5.0022839

[42] Xiao, J., Hu, R. and Chen, G. 2020. Micro-nano-engineered nitrogenous bone biochar developed with a ball-milling technique for high-efficiency removal of aquatic $\mathrm{Cd}(\mathrm{II})$, $\mathrm{Cu}(\mathrm{II})$ and $\mathrm{Pb}$ (II). Journal of Hazardous Materials. 387: 121980. DOl:https://doi.org/10.1016/j.jhazmat.2019.121980

[43] Park, J.-H., Yun, J.-J., Kang, S.-W., Kim, S.-H., Cho, J.-S., Wang, J.J. \& Seo, D.-C. 2021. Removal of potentially toxic metal by biochar derived from rendered solid residue with high content of protein and bone tissue. Ecotoxicology and Environmental Safety. 208: 111690.

DOI: https://doi.org/10.1016/j.ecoenv.2020.111690

[44] Barambu, N.U., Bilad, M.R., Bustam, M.A. , Kurnia, K.A., Othman, M.H.D., Nordin, N.A.H.M. 2020. Development of membrane material for oily wastewater treatment: A review. Ain Shams Engineering Journal. 12 (2): 1361-1376.

DOI: https://doi.org/10.1016/j.asej.2020.08.027

[45] Martini, S. and Yuliwati, E. 2020. Membrane Development and Its Hybrid Application for Oily Wastewater Treatment: A Review, Journal of Applied Membrane Science \& Technology, 25(1): 57-71. DOI: https://doi.org/10.11113/amst.v25n1.209

[46] Khalifa, O., Banat, F., Srinivasakannan, C., AlMarzooqi, F., \& Hasan, S.W. 2020. Ozonation-assisted electro-membrane hybrid reactor for oily wastewater treatment: A methodological approach and synergy effects. Journal of Cleaner Production. 289: 125764. DOI: https://doi.org/10.1016/j.jclepro.2020.125764

[47] Bakar, A.A., Mazlan, W.W., Akbar, N., Badrealam, S. \& Ali, K. M. 2019. Agriculture waste from banana peel as low cost adsorbent in treating methylene blue from batik textile waste water effluents, in: Journal of Physics: Conference Series. 1349: 012078.

DOI: https://doi:10.1088/1742-6596/1349/1/012078

[48] Bansal, M., Patnala, P.K. and Dugmore, T. 2020. Adsorption of Eriochrome Black-T(EBT) using tea waste as a low cost adsorbent by batch studies: A green approach for dye effluent treatments. Current Research in Green and Sustainable Chemistry. 3: 100036. DOI: https://doi.org/10.1016/j.crgsc.2020.100036

[49] Tapfuma, A., Chakawa, D. P., Moyo, L., Hlabangana, N., Danha, G. \& Muzenda, E. 2019. Investigating the feasibility 
of using Agricultural Waste as an Adsorbent of Gold lons in Small Scale Gold Processing Plants. Procedia Manufacturing. 35: 85-90.

DOI: https://doi.org/10.1016/j.promfg.2019.05.008.

[50] Niazi, N. K., Bibi, I., Shahid, M., Ok, Y. S., Shaheen, S. M., Rinklebe, J., Wang, H., Murtaza, B., Islam, E. \& Nawaz, M. F. 2018. Arsenic Removal by Japanese Oak Wood Biochar in Aqueous Solutions and Well Water: Investigating Arsenic Fate using Integrated Spectroscopic and Microscopic Techniques. Science of the Total Environment. 621: 16421651. DOI: https://doi.org/10.1016/j.scitotenv.2017.10.063.

[51] Shaheen, S. M., Niazi, N. K., Hassan, N. E., Bibi, I., Wang, H., Tsang, D. C., Ok, Y. S., Bolan, N. \& Rinklebe, J. 2019. Woodbased Biochar for the Removal of Potentially Toxic Elements in Water and Wastewater: A Critical Review, International Materials Reviews. 64(4): 216-247. DOI: https://doi.org/10.1080/09506608.2018.1473096.

[52] Hagos, M., Yimam, A. \& Gebru, K. A. 2020. Removal of Cu (II) ion from Ezana (Meli) Gold Mining Wastewater using Eucalyptus Bark as Adsorbent, (2020). Research Square. Access: 1 April 2021.

DOI:https://assets.researchsquare.com/files/rs103759/v1_stamped.pdf.

[53] Cemin, A., Ferrarini, F., Poletto, M., Bonetto, L. R., Bortoluz, J., Lemée, L., Guégan, R., Esteves, V. I. \& Giovanela, M. 2021. Characterization and Use of a Lignin Sample Extracted from Eucalyptus Grandis Sawdust for the Removal of Methylene Blue Dye. International Journal of Biological Macromolecules. 170: 375-389. DOI: https://doi.org/10.1016/j.ijbiomac.2020.12.155.

[54] Abatal, M., Anastopoulos, I., Giannakoudakis, D. A. \& Olguin, M. T. 2020. Carbonaceous Material Obtained from Bark Biomass as Adsorbent of Phenolic Compounds from Aqueous Solutions. Journal of Environmental Chemical Engineering. 8(2020): 103784 DOl:https://doi.org/10.1016/j.jece.2020.10378.

[55] Samaraweera, H., Pittman, C. U., Thirumalai, R. V. K. G., Hassan, E. B., Perez, F. \& Mlsna, T. 2021. Characterization of Graphene/pine Wood Biochar Hybrids: Potential to Remove Aqueous Cu2+. Environmental Research. 192: 110283. DOI: https://doi.org/10.1016/j.envres.2020.1 10283.

[56] Zhang, R. and Leiviskä, T. 2020. Surface Modification of Pine Bark with Quaternary Ammonium Groups and Its Use for Vanadium Removal. Chemical Engineering Journal. 385: 123967. DOI: https://doi.org/10.1016/j.cej.2019.123967.

[57] Wierzbaa, S., Dołhańczuk-Śródkaa, A. and Křǐžb, J. 2020. Optimization of the Sorption Process of Copper Cations from Aqueous Solution by Pine Bark (Pinus sylvestris). Desalination and Water Treatment. 186: 258-266.

DOI: https://doi: 10.5004/dwt.2020.25626.

[58] Zhang, P., O'Connor, D., Wang, Y., Jiang, L., Xia, T., Wang, L.., Tsang, D. C., OK, Y. S. \& Hou, D. 2020. A Green Biochar/iron Oxide Composite for Methylene Blue Removal. Journal of Hazardous Materials. 384: 121286. DOI: https://doi.org/10.1016/j.jhazmat.2019.121286.

[59] Ashraf, A., Bibi, I., Niazi, N. K., Ok, Y. S., Murtaza, G., Shahid, M., Kunhikrishnan, A., Li, D. \& Mahmood, T. 2017. Chromium (VI) Sorption Efficiency of Acid-activated Banana Peel Over Organo-montmorillonite in Aqueous Solutions. International Journal of Phytoremediation. 19(7): 605-613. DOI: https://doi.org/10.1080/15226514.2016.1256372.

[60] Li, H., Xiong, J., Xiao, T., Long, J., Wang, Q., Li, K., Liu, X., Zhang, G. \& Zhang, H. 2019. Biochar Derived from Watermelon Rinds as Regenerable Adsorbent for Efficient Removal of Thallium(I) from Wastewater. Process Safety and Environmental Protection. 127: 257-266. DOl: https://doi.org/10.1016/j.psep.2019.04.031.

[61] Shakoor, M. B., Niazi, N. K., Bibi, I., Shahid, M., Sharif, F., Bashir, S., Shaheen, S. M., Wang, H., Tsang, D. C. \& Ok, Y. S. 2018. Arsenic Removal by Natural and Chemically Modified Water Melon Rind in Aqueous Solutions and
Groundwater. Science of the Total Environment. 645: 14441455.

DOI: https://doi.org/10.1016/j.scitotenv.2018.07.218.

[62] Devasangeeth, S. D., Balaji, G. and Lakshmipathy, R. 2018. Multi Metal Ion Sorption Capacity of Watermelon Rind Extract Capped ZnS Nanoparticles. International Journal of Pure and Applied Mathematics. 118(24). DOI: https://www.acadpubl.eu/hub/.

[63] Daffalla, S. B., Mukhtar, H. \& Shaharun, M. S. 2020. Preparation and Characterization of Rice Husk Adsorbents for Phenol Removal from Aqueous Systems. PloS one. 15(12): e0243540.

DOl: https://doi.org/10.1371/journal.pone.0243540.

[64] Bhatti, H. N., Safa, Y., Yakout, S. M., Shair, O. H., Iabal, M. \& Nazir, A. 2020. Efficient Removal of Dyes Using Carboxymethyl Cellulose/alginate/polyvinyl Alcohol/rice Husk Composite: Adsorption/desorption, Kinetics and Recycling Studies. International Journal of Biological Macromolecules. 150: 861-870. DOI: https://doi.org/10.1016/j.ijbiomac.2020.02.093.

[65] Fu, Y., Shen, Y., Zhang, Z., Ge, X. \& Chen, M. 2019. Activated Bio-chars Derived from Rice Husk via One- and Two-step $\mathrm{KOH}$-catalyzed Pyrolysis for Phenol Adsorption. Science of The Total Environment. 646: 1567-1577. DOI: https://doi.org/10.1016/j.scitotenv.2018.07.423.

[66] Jiang, X., Sun, P., Xu, L., Xue, Y., Zhang, H. \& Zhu, W. 2020. Platanus Orientalis Leaves based Hierarchical Porous Carbon Microspheres as High Efficiency Adsorbents for Organic Dyes Removal. Chinese Journal of Chemical Engineering. 28(1): 254-265. DOl: https://doi.org/10.1016/j.cjche.2019.03.030.

[67] Guo, D., Li, Y., Cui, B., Hu, M., Luo, S., Ji, B. \& Liu, Y. 2020. Natural Adsorption of Methylene Blue by Waste Fallen Leaves of Magnoliaceae and Its Repeated Thermal Regeneration for Reuse. Journal of Cleaner Production. 267: 121903 .

DOI: https://doi.org/10.1016/j.jclepro.2020.121903.

[68] Denisova, T. R. 2018. Study of Phenol Adsorption by Modified Birch Leaves: Preparation and Adsorption Characteristics. International Journal of Green Pharmacy (IJGP). 11 (4): 8872 - 8876. DOI: https://doi.org/10.22377/ijgp.v1 1i04.1418.

[69] Mandal, A., Bar, N. \& Das, S. K. 2020. Phenol Removal from Wastewater using Low-cost Natural Bioadsorbent Neem (Azadirachta Indica) Leaves: Adsorption Study and MLR Modeling. Sustainable Chemistry and Pharmacy. 17: 100308. DOI: https://doi.org/10.1016/j.scp.2020.100308.

[70] Malakootian, M., Jafari Mansoorian, H., Alizadeh, M. \& Baghbanian, A. 2017. Phenol Removal from Aqueous Solution by Adsorption Process: Study of The Nanoparticles Performance Prepared from Alo vera and Mesquite (Prosopis) Leaves. Scientia Iranica. 24(6): 3041-3052. DOl:https://doi.org/10.24200/SCl.2017.4524.

[71] Bediako, J. K., Lin, S., Sarkar, A. K., Zhao, Y., Choi, J.-W., Song, M.-H., Cho, C.-W. \& Yun, Y.-S. 2020. Evaluation of Orange Peel-derived Activated Carbons for Treatment of Dye-contaminated Wastewater Tailings. Environmental Science and Pollution Research. 27(1): 1053-1068. DOI: https://doi.org/10.1007/s1 1356-019-07031-8.

[72] Bibaj, E., Lysigaki, K., Nolan, J., Seyedsalehi, M., Deliyanni, E., Mitropoulos, A. \& Kyzas, G. Activated Carbons from Banana Peels for the Removal of Nickel Ions. International Journal of Environmental Science and Technology. 16(2): 667-680.

DOI: https://doi.org/10.1007/s13762-018-1676-0.

[73] Abid, M. K., Ibrahim, H. B. \& Zulkifli, S. Z. 2019. Synthesis and Characterization of Biochar from Peel and Seed of Jackfruit Plant Waste for the Adsorption of Copper Metal Ion from Water. Research Journal of Pharmacy and Technology. 12(2): 4182-4188. DOI: https://doi: 10.5958/0974-360X.2019.00720.0.

[74] Ranasinghe, S., Navaratne, A., Priyantha, N. 2018. Enhancement of Adsorption Characteristics of $\mathrm{Cr}$ (III) and 
Ni (II) by Surface Modification of Jackfruit Peel Biosorbent, Journal of Environmental Chemical Engineering. 6(5): 56705682.

DOI:https://doi.org/10.1016/j.jece.2018.08.058.

[75] Kooh, M. R. R., Dahri, M. K. \& Lim, L. B. L. 2018. Jackfruit Seed as Low-cost Adsorbent for Removal of Malachite Green: Artificial Neural Network and Random Forest Approaches. Environmental Earth Sciences. 77(12): 434. DOl: https://doi.org/10.1007/s12665-018-7618-9.

[76] Dai, H., Huang, Y., Zhang, Y., Zhang, H. \& Huang, H. 2019. Green and Facile Fabrication of Pineapple Peel Cellulose/Magnetic Diatomite Hydrogels in Ionic Liquid for Methylene Blue Adsorption. Cellulose. 26(6): 3825-3844. DOI: https://doi.org/10.1007/s10570-019-02283-6.

[77] Ngabura, M., Hussain, S. A., Ghani, W. A. W., Jami, M. S. \& Tan, Y. P. 2018. Utilization of Renewable Durian Peels for Biosorption of Zinc from Wastewater. Journal of Environmental Chemical Engineering. 6(2): 2528-2539. DOI: https://doi.org/10.1016/j.jece.2018.03.052.

[78] Sathiyavimal, S., Vasantharaj, S., Shanmugavel, M., Manikandan, E., Nguyen-Tri, P., Brindhadevi, K. \& Pugazhendhi, A. 2020. Facile Synthesis and Characterization of Hydroxyapatite from Fish Bones: Photocatalytic Degradation of Industrial Dyes (Crystal Violet and Congo Red). Progress in Organic Coatings. 148(2020): 105890.

DOI: https://doi.org/10.1016/j.porgcoat.2020.105890.

[79] Park, J. H., Wang, J. J., Kim, S. H., Kang, S. W., Cho, J. S., Delaune, R. D., Ok, Y. S. \& Seo, D. C. Lead Sorption Characteristics of Various Chicken Bone Part-derived Chars. Environmental Geochemistry and Health. 41(4): 1675-1685 DOI: https://doi.org/10.1007/s10653-017-0067-7.

[80] Alquzweeni, S. S. and Alkizwini, R. S. 2020. Removal of Cadmium from Contaminated Water Using Coated Chicken Bones with Double-Layer Hydroxide (Mg/Fe-LDH). Water. 12(8): 2303.

DOI: https://doi.org/10.3390/w12082303.

[81] Côrtes, L. N., Druzian, S. P., Streit, A. F. M., Junior, T. R. S. a. C., Collazzo, G. C. \& Dotto, G. L. 2019. Preparation of Carbonaceous Materials from Pyrolysis of Chicken Bones and Its Application for Fuchsine Adsorption. Environmental Science and Pollution Research. 26(28): 28574-28583. DOI: https://doi.org/ 10.1007/s1 1356-018-3679-2.

[82] Medellín-Castillo, N. A., Cruz-Briano, S. A., Leyva-Ramos, R., Moreno-Piraján, J. C., Torres-Dosal, A., Giraldo-Gutiérrez, L., Labrada-Delgado, G. J., Pérez, R. O., Rodriguez-Estupiñan, S. Y. Reyes Lopez, M. S. Berber Mendoza, J. P. 2020. Use of Bone Char Prepared from an Invasive Species, Pleco Fish (Pterygoplichthys spp.), to Remove Fluoride and Cadmium(II) in Water. Journal of Environmental Management. 256: 109956.2 DOI: https://doi.org/10.1016/j.jenvman.2019.109956.

[83] C. F. Okey-Onyesolu, O. D. Onukwuli, M. I. Ejimofor, C. C. Okoye. Kinetics and Mechanistic Analysis of Particles Decontamination from Abattoir Wastewater ( $A B W)$ using Novel Fish Bone Chito-protein (FBC). Heliyon. 6(8): e04468. DOI: https://doi.org/10.1016/j.heliyon.2020.e04468.

[84] Módenes, A. N., Bazarin, G., Borba, C. E., Locatelli, P. P. P., Borsato, F. P., Pagno, V., Pedrini, R., Trigueros, D. E. G., Espinoza-Quiñones, F. R. \& Scheufele, F. B. 2021. Tetracycline Adsorption by Tilapia Fish Bone-based Biochar: Mass Transfer Assessment and Fixed-bed Data Prediction by Hybrid Statistical-phenomenological Modeling. Journal of Cleaner Production. 279(2021): 123775. DOI: https://doi.org/10.1016/j.jclepro.2020.123775.

[85] Simpen, I. N., Negara, I. M. S. \& Jayanto, S. D. 2020. Activated Adsorbent Prepared from Bovine Bone Waste: Physico-chemical Characteristics, Isoterm and Thermodynamics Adsorption of Chromium lons in Wastewater. International Journal of Engineering Technologies and Management Research. 7(3): 1-11. DOI: https://doi.org/10.29121/ijetmr.v7.i3.2020.534
[86] Maldonado, H. J. A., Torres García, F. A., Salazar Hernández, M. M. and Hernández Soto, R. 2017. Removal of Chromium from Contaminated Liquid Effluents using Natural Brushite Obtained from Bovine Bone. Desalination and Water Treatment. 95: 262-273. DOI: https://doi: 10.5004/dwt.2017.21480.

[87] Wang, M. Liu, Y., Yao, Y., Han, L. \& Liu, X. 2020. Comparative Evaluation of Bone Chars Derived from Bovine Parts: Physicochemical Properties and Copper Sorption Behavior. Science of the Total Environment. 700: 134470. https://doi.org/10.1016/j.scitotenv.2019.134470.

[88] Olaoye, R. A., Afolayan, O. D., Adeyemi, K. A., Ajisope, L. O. \& Adekunle, O. S. 2020. Adsorption of Selected Metals from Cassava Processing Wastewater Using Cow-bone Ash. Scientific African. 10: e00653. DOl: https://doi.org/10.1016/j.sciaf.2020.e00653.

[89] Malla, K. P., Adhikari, R., JeewanYadav, R., Nepal, A. \& Neupane, B. P. 2018. Removal of Lead (II) Ions from Aqueous Solution by Hydroxyapatite Biosorbent Extracted from Ostrich Bone. Journal of Health and Allied Sciences. 7(1): 27-33. .DOI: https://doi.org/10.37107/jhas.19

[90] Amiri, M. J., Abedi-Koupai, J. \& Eslamian, S. 2017. Adsorption of $\mathrm{Hg}$ (II) and $\mathrm{Pb}$ (II) lons by Nanoscale Zero Valent Iron Supported on Ostrich Bone Ash in a Fixed-bed Column System. Water Science and Technology. 76(3): 671-682.

DOI: https://doi.org/10.2166/wst.2017.252.

[91] Gil, A., Amiri, M. J., Abedi-Koupai, J. \& Eslamian, S. 2018. Adsorption/reduction of $\mathrm{Hg}$ (II) and $\mathrm{Pb}$ (II) from Aqueous Solutions by using Bone Ash/nZVI Composite: Effects of Aging Time, Fe Loading Quantity and Co-existing lons. Environmental Science and Pollution Research. 25(3): 28142829. DOI: https://doi.org/10.1007/s1 1356-017-0508-y.

[92] Rashed, M. N., Gad, N. M. \& Fathy, A. A. -E. 2019. Adsorption of $\mathrm{Cd}$ (II) and $\mathrm{Pb}$ (II) Using Physically Pretreated Camel Bone Biochar. Advanced Journal of ChemistrySection A. 2(4): 347-364 DOl:https://doi.org/10.33945/SAMI/AJCA.2019.4.8.

[93] Alqadami, A. A., Khan, M. A., Otero, M., Siddiqui, M. R., Jeon, B.-H. \& Batoo, K. M. 2018. A Magnetic Nanocomposite Produced from Camel Bones for an Efficient Adsorption of Toxic Metals from Water. Journal of Cleaner Production. 178(2018): 293-304. DOl: https://doi.org/10.1016/j.jclepro.2018.01.023.

[94] Abd-Rabboh, H. S., Fawy, K. F. \& Awwad, N. S. 2019. Removal of Copper (II) from Aqueous Samples using Natural Activated Hydroxyapatite Sorbent Produced from Camel Bones. Desalination and Water Treatment. 164: 300309. DOI: https://doi:10.5004/dwt.2019.24371.

[95] Zhou, Y., Chang, D. \& Chang, J. 2017. Preparation of Nano-structured Pig Bone Hydroxyapatite for High-efficiency Adsorption of $\mathrm{Pb} 2+$ from Aqueous Solution. International Journal of Applied Ceramic Technology. 14(6): 1125-1133. DOI: https://doi.org/10.1111/ijac.12749.

[96] Sekine, Y., Nankawa, T., Yamada, T., Matsumura, D., Nemoto, Y., Takeguchi, M., Sugita, T., Shimoyama, I., Kozai, N. \& Morooka, S. 2021. Carbonated Nanohydroxyapatite from Bone Waste and Its Potential as a Super Adsorbent for Removal of Toxic lons. Journal of Environmental Chemical Engineering. 9(2): $105114 . \quad$ DOI: https://doi.org/10.1016/j.jece.2021.105114.

[97] Chakraborty, R., Asthana, A., Singh, A. K., Verma, R., Sankarasubramanian, S., Yadav, S., Carabineiro, S. A. C. \& Susan, M. A. B. H. 2020. Chicken Feathers Derived Materials for the Removal of Chromium from Aqueous Solutions: Kinetics, Isotherms, Thermodynamics and Regeneration Studies. Journal of Dispersion Science and Technology. 115. DOI: https://doi.org/10.1080/01932691.2020.1842760.

[98] Sun, P., Zhu, G., Li, T., Li, X., Shi, Q., Xue, M. \& Li, B. 2020. Acidification Chicken Feather as Sorbent for Selectively Achicken Feather as Sorbent for Selectively Adsorbing of 
$\mathrm{Cr}(\mathrm{VI})$ Ions in Aqueous Solution. Materials Today Communications. 24: 101358.

DOI: https://doi.org/10.1016/j.mtcomm.2020.101358.

[99] Nasiebanda, R., Wambu, E.W. \& Lusweti, K. 2021. Water Defluoridation by Adsorption Using Aluminium Modified Chicken Feathers. African Journal of Education, Science and Technology. 6(2): 278-291. DOI: http://ajest.info/index.php/ajest/article/view/518.

[100] Foroutan, R., Peighambardoust, S. J., Hosseini, S. S., Akbari, A. \& Ramavandi, B. 2021. Hydroxyapatite Biomaterial Production from Chicken (Femur and Beak) and Fishbone Waste through a Chemical Less Method for Cd2+ Removal from Shipbuilding Wastewater. Journal of Hazardous Materials. 413:

125428. DOl:https://doi.org/10.1016/j.jhazmat.2021.125428.

[101] Wang, H., Lv, Z., Wang, Y.-n., Sun, Y. \& Tsang, Y. F. 2021. Recycling of Biogenic Hydroxyapatite (HAP) for Cleaning of Lead from Wastewater: Performance and Mechanism. Environmental Science and Pollution Research. 28: 2950929520. DOI: https://doi.org/10.1007/s1 1356-020-10855-4.

[102] Saraswat, S. K., Demir, M. \& Gosu, V. 2020. Adsorptive Removal of Heavy Metals from Industrial Effluents using
Cow Dung as the Biosorbent: Kinetic and Isotherm Modeling. Environmental Quality Management. 30(1): 5160. DOI: https://doi.org/10.1002/tqem.21703.

[103] Henrique, D. C., Quintela, D. U., Ide, A. H., Erto, A., Duarte, J. L. d. S. \& Meili, L. 2020. Calcined Mytella Falcata Shells as Alternative Adsorbent for Efficient Removal of Rifampicin Antibiotic from Aqueous Solutions. Journal of Environmental Chemical Engineering. 8(3): 103782. DOI: https://doi.org/10.1016/j.jece.2020.103782.

[104] Khan, N., Chowdhary, P., Ahmad, A., Shekher Giri, B. \& Chaturvedi, P. 2020. Hydrothermal Liquefaction of Rice Husk and Cow Dung in Mixed-Bed-Rotating Pyrolyzer and Application of Biochar for Dye Removal. Bioresource Technology. 309: 123294.

DOI:https://doi.org/10.1016/j.biortech.2020.123294.

[105] Pawar, S. and Theodore, T. 2020. Development of Hydroxyapatite from Waste Mutton Bones and Its Application for Hexavalent Chromium Removal from Aqueous Solutions-adsorption Isotherms and Kinetics Study. AIP Conference Proceedings. AIP Publishing LLC. 030001. DOI: https://doi.org/10.1063/5.0022839. 